\title{
Quasi-static Planar Deformation in a Medium Composed of Elastic and Thermoelastic Solid Half Spaces Due to Seismic Sources in an Elastic Solid
}

\author{
Anil K. VASHISTH ${ }^{1}$, Kavita RANI ${ }^{2}$, and Kuldip SINGH ${ }^{3}$ \\ ${ }^{1}$ Department of Mathematics, Kurukshetra University, Kurukshetra, India \\ e-mail: akvashishth@kuk.ac.in (corresponding author) \\ ${ }^{2}$ Department of Mathematics, Government Post Graduate College, Hisar, India \\ e-mail: karya4@gmail.com \\ ${ }^{3}$ Department of Mathematics, Guru Jambheshwar University of Science \\ and Technology, Hisar, India; e-mail: profkbgju@gmail.com
}

\begin{abstract}
A two-dimensional problem of quasi static deformation of a medium consisting of an elastic half space in welded contact with thermoelastic half space, caused due to seismic sources, is studied. Source is considered to be in the elastic half space. The basic equations, governed by the coupled theory of thermoelasticity, are used to model for thermoelastic half space. The analytical expressions for displacements, strain and stresses in the two half spaces are obtained first for line source and then for dip slip fault. The results for two particular cases, adiabatic conditions and isothermal conditions, are also obtained. Numerical results for displacements, stresses and temperature distribution have also been computed and are shown.
\end{abstract}

Key words: seismic sources, thermoelastic, quasi-static, deformation.

Ownership: Institute of Geophysics, Polish Academy of Sciences;

(C) 2015 Vashisth et al. This is an open access article distributed under the Creative Commons Attribution-NonCommercial-NoDerivs license,

http://creativecommons.org/licenses/by-nc-nd/3.0/. 


\section{INTRODUCTION}

The elasticity theory of dislocation was developed and applied by Steketee (1958), Rongved and Frasier (1958), and Maruyama (1964, 1966). The problems related to seismic sources in elastic media have been studied extensively by many researchers (Burridge and Knopoff 1964, Singh and BenMenahem 1969, Singh 1970, Sato 1971, Singh et al. 1973, Sato and Matsu'ura 1973, Jovanovich et al. 1974a, b; Freund and Barnett 1976, etc.). The detailed description about seismic sources is given in the classical texts: Aki and Richards (1980), Ben-Menahem and Singh (1981), Lay and Wallace (1995), and Stein and Wysession (2003).

Singh and Garg (1985) studied the static deformation of an isotropic multilayered half space by a normal line load and a shear line load. Singh and Garg (1986) described the representation of two-dimensional seismic sources and obtained the integral expressions for the Airy stress function in an unbounded medium due to various two-dimensional sources and represented the sources in terms of jumps across the plane through the sources. Garg and Singh (1987) extended the results of Singh and Garg (1985) by considering the multilayered half space as transversely isotropic. Pan $(1989 a, b)$ provided a unified solution of the static deformation of the transversely isotropic and layered half space by general surface loads. Rani et al. (1991) extended the work of Singh and Garg (1986) and obtained closed form analytical expressions for the displacements and stresses at any point of a uniform half space due to two-dimensional buried sources by applying the traction free boundary conditions at the surface of the half space.

Okada $(1985,1992)$ provided compact analytical expressions for the surface deformation and internal deformation due to inclined shear and tensile faults in a homogeneous isotropic half space. Heaton and Heaton (1989) obtained the deformation field induced by point forces and point force couples embedded in two Poissonian half spaces in welded contact. Singh et al. (1992) derived closed form expressions for displacements and stresses in two welded half spaces caused by two-dimensional sources. Many other researchers discussed source problems for different types of sources viz. Kumari et al. (1992), Singh et al. (1993, 2003), Garg et al. (1996, 2003), Tomar and Dhiman (2003), Kumar et al. (2005), Singh et al. (2005), and Madan et al. (2005).

Thermoelasticity deals with dynamical systems whose interactions with the surroundings include not only mechanical work and external work but also the exchange of heat. Theory of thermoelasticity studies the influence of temperature of an elastic medium on the distribution of stress and strain as well as the inverse effect of the deformation on the temperature distribution. 
Attempts have been made to study source problems in thermoelasticity. However, most of such studies are attributed to internal and surface heat sources, (e.g., Lanzano 1986a,b, Dziewonski and Anderson 1981, Rundle 1982, Small and Booker 1986, Abd-Alla 1995, Shevchenko and Gol'tsev 2001, Kit et al. 2001, Youssef 2006, 2009, 2010, Mallik and Kanoria 2008, Hou et al. 2008a, b, 2009, 2011, Kumar and Gupta 2009, Attetkov et al. 2009 , etc.). Some authors have considered mechanical sources also; e.g., Pan (1990) considered quasi-static governing equations of thermoelasticity and discussed the transient thermoelastic deformation in a transversely isotropic and layered half space by surface loads and internal sources. Kumar and Rani (2004) considered a dynamical two-dimensional problem of thermoelasticity and studied the deformation due to mechanical and thermal sources in generalized thermally conducting orthorhombic material. Ghosh and Kanoria (2007) derived analytical expressions for thermoelastic displacements and stresses in composite multi-layered media due to varying temperature and concentrated loads. Quasi-static deformation of a thermoelastic medium due to seismic sources or quasi static mechanical sources has not been studied so far.

For a realistic Earth model, it is appropriate to involve thermoelastic medium in the model. The study of quasi static deformation of a thermo- elastic medium, in welded contact with an elastic medium, due to seismic sources is important for its geophysical applications. The theory developed in this paper may find its applications in seismic faulting. When the source surface is very long in one dimension in comparison with the other, the use of twodimensional approximations is justified and consequently calculations are simplified to a great extent and one gets a closed form of analytic solution. A very long strip source and a very long line source are the examples of such two-dimensional sources.

In this paper, quasi static deformation of a medium consisting of a homogeneous isotropic thermoelastic half space in welded contact with a homogeneous elastic half space, due to a line source and dip slip fault in an elastic half space, is studied. Numerical results for displacements, stresses and temperature distribution are presented graphically. The present problem is useful in the field of geomechanics where the interest is about the various phenomena occurring in the earthquakes and measuring of displacements, stresses, and temperature field due to the presence of certain sources.

\section{FORMULATION OF THE PROBLEM}

Consider a medium consisting of thermoelastic half spaces $(z \geq 0)$ and an elastic half space $(z \leq 0)$ which are in welded contact along the plane $z=0$, as shown in Fig. 1. A line source parallel to the $x$-axis passing through the point $(0,0,-h)$ in the elastic half space is considered. 


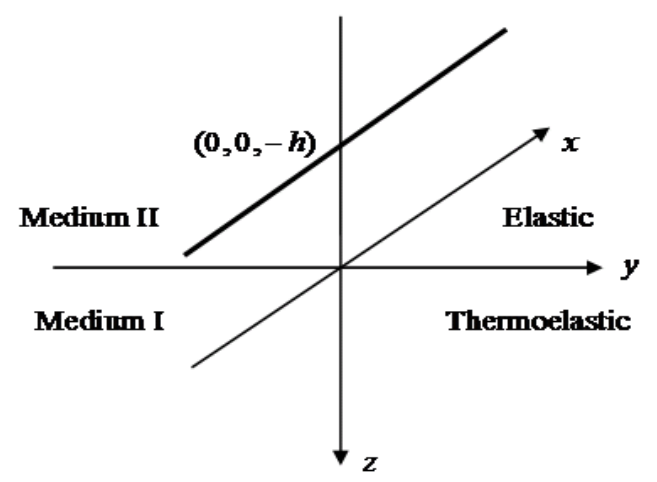

Fig. 1. A line source through the point $(0,0,-h)$.

A two-dimensional plane strain problem in $y z$-plane is considered so that the displacement components can be written as:

$$
u_{i}=u_{i}(y, z, t), \quad(i=y, z), \quad u_{x}=0 .
$$

\section{BASIC EQUATIONS AND THEIR SOLUTIONS}

\subsection{For thermoelastic half space}

The stress strain relations for a thermoelastic medium (Nowacki 1975) are given by:

$$
\sigma_{i j}=\lambda \varepsilon_{k k} \delta_{i j}+2 \mu \varepsilon_{i j}-\beta \theta \delta_{i j}, \quad i, j, k=x, y, z,
$$

where $\sigma_{j i}$ and $\varepsilon_{i j}$ are components of stress and strain tensor, respectively, $\lambda, \mu$ are Lame's constants, $\beta=(3 \lambda+2 \mu) \alpha_{t}$ is the thermoelastic coupling coefficient, $\theta$ is the temperature difference, and $\alpha_{t}$ is the coefficient of linear thermal expansion.

The stress components for a plane strain problem in the $y z$-plane are given by:

$$
\begin{gathered}
\sigma_{y y}=\lambda \varepsilon_{k k}+2 \mu \varepsilon_{y y}-\beta \theta, \\
\sigma_{z z}=\lambda \varepsilon_{k k}+2 \mu \varepsilon_{z z}-\beta \theta, \\
\sigma_{y z}=2 \mu \varepsilon_{y z},
\end{gathered}
$$

where

$$
\varepsilon_{k k}=\frac{1}{2(\lambda+\mu)}\left(\sigma_{y y}+\sigma_{z z}+2 \beta \theta\right) .
$$

The strain components can be represented as: 


$$
\begin{gathered}
2 \mu \varepsilon_{y y}=(1-v) \sigma_{y y}-v \sigma_{z z}+\alpha_{0} \theta, \\
2 \mu \varepsilon_{z z}=(1-v) \sigma_{z z}-v \sigma_{y y}+\alpha_{0} \theta, \\
2 \mu \varepsilon_{y z}=\sigma_{y z},
\end{gathered}
$$

where $v=\frac{\lambda}{2(\lambda+\mu)}$ is Poisson's ratio and $\alpha_{0}=(1-2 v) \beta$.

The equations of equilibrium for thermoelastic medium, in the absence of body forces, are

$$
\begin{aligned}
& \sigma_{y y, y}+\sigma_{y z, z}=0, \\
& \sigma_{z y, y}+\sigma_{z z, z}=0,
\end{aligned}
$$

and the compatibility equation is

$$
\varepsilon_{y y, z z}+\varepsilon_{z z, y y}=2 \varepsilon_{y z, y z} .
$$

Using Eqs. 4 and 5 in Eq. 6, we get

$$
\nabla^{2}\left(\sigma_{y y}+\sigma_{z z}+2 \eta \theta\right)=0
$$

where

$$
\eta=\frac{\alpha_{0}}{2(1-v)}=\frac{(1-2 v)}{2(1-v)} \beta, \quad \nabla^{2} \equiv \frac{\partial^{2}}{\partial y^{2}}+\frac{\partial^{2}}{\partial z^{2}}
$$

The heat conduction equation can be written as

$$
\lambda_{0} \theta_{, i i}-\rho C_{e} \dot{\theta}-\beta T_{0} \dot{\varepsilon}_{k k}=0, \quad(i, k=y, z),
$$

where $\lambda_{0}$ is the thermal conductivity, $C_{e}$ is the specific heat, $\rho$ is the density, and $T_{0}$ is the temperature at natural state.

The stress function $U$ is defined as:

$$
\sigma_{y y}=\frac{\partial^{2} U}{\partial z^{2}}, \quad \sigma_{z z}=\frac{\partial^{2} U}{\partial y^{2}}, \quad \sigma_{y z}=-\frac{\partial^{2} U}{\partial y \partial z} .
$$

Using Eqs. 3d and 9 in Eqs. 7 and 8, we get

$$
\nabla^{2}\left(\nabla^{2} U+2 \eta \theta\right)=0
$$

and

$$
\lambda_{0} \nabla^{2} \theta-\left(\rho C_{e}+\frac{\beta^{2} T_{0}}{(\lambda+\mu)}\right) \dot{\theta}-\frac{\beta T_{0}}{2(\lambda+\mu)}\left(\nabla^{2} \dot{U}\right)=0 .
$$

Equations 10 and 11 imply that

$$
\left(c \nabla^{2}-\frac{\partial}{\partial t}\right) \nabla^{2} \theta=0
$$


and

$$
\left(c \nabla^{2}-\frac{\partial}{\partial t}\right) \nabla^{4} U=0
$$

where

$$
c=\lambda_{0}\left[\rho C_{e}+\frac{\alpha_{0}^{2} T_{0}}{\mu(1-2 v)}-\frac{\alpha_{0}^{2} T_{0}}{2 \mu(1-v)}\right]^{-1} .
$$

The general solution of Eq. 12 may be written as

$$
\theta=\theta_{1}+\theta_{2},
$$

where

$$
c \nabla^{2} \theta_{1}=\frac{\partial \theta_{1}}{\partial t}
$$

and

$$
\nabla^{2} \theta_{2}=0
$$

Similarly, the general solution of Eq. 13 can be expressed as:

$$
U=U_{1}+U_{2},
$$

where

$$
c \nabla^{2} U_{1}=\frac{\partial U_{1}}{\partial t}
$$

and

$$
\nabla^{4} U_{2}=0
$$

Equations 16, 17, 19, and 20, with the time dependence as $e^{-i \omega t}$, can be written as

$$
\begin{gathered}
\nabla^{2} \theta_{1}+\frac{i \omega}{c} \theta_{1}=0, \\
\nabla^{2} \theta_{2}=0, \\
\nabla^{2} U_{1}+\frac{i \omega}{c} U_{1}=0, \\
\nabla^{4} U_{2}=0,
\end{gathered}
$$

where $\theta_{1}, \theta_{2}, U_{1}$, and $U_{2}$ are functions of $y$ and $z$ only.

Application of Fourier transform to Eqs. 21-24, solution of the resulting differential equations, inversion of Fourier transform and further simplification leads to

$$
\theta=\int_{0}^{\infty}\left(A_{1} e^{-m z}+A_{2} e^{-k z}\right)\left(\begin{array}{c}
\sin k y \\
\cos k y
\end{array}\right) d k,
$$




$$
U=\int_{0}^{\infty}\left(B_{1} e^{-m z}+\left(B_{2}+B_{3} k z\right) e^{-k z}\right)\left(\begin{array}{c}
\sin k y \\
\cos k y
\end{array}\right) d k
$$

where

$$
m=\left(k^{2}-\frac{i \omega}{c}\right)^{1 / 2}, \quad \operatorname{Re}(m)>0
$$

and $A_{i}, B_{i}$ may be functions of $k$. Equation 9 gives

$$
\begin{aligned}
& \sigma_{y y}=\int_{0}^{\infty}\left(m^{2} B_{1} e^{-m z}+\left(B_{2} k^{2}-2 B_{3} k^{2}+B_{3} k^{3} z\right) e^{-k z}\right)\left(\begin{array}{c}
\sin k y \\
\cos k y
\end{array}\right) d k, \\
& \sigma_{z z}=\int_{0}^{\infty}\left(B_{1} e^{-m z}+\left(B_{2}+B_{3} k z\right) e^{-k z}\right)\left(\begin{array}{c}
\sin k y \\
\cos k y
\end{array}\right)\left(-k^{2}\right) d k, \\
& \sigma_{y z}=\int_{0}^{\infty}\left(m B_{1} e^{-m z}+\left(\left(B_{2}-B_{3}\right) k+B_{3} k^{2} z\right) e^{-k z}\right) k\left(\begin{array}{c}
\cos k y \\
-\sin k y
\end{array}\right) d k .
\end{aligned}
$$

Making use of Eqs. 25 and 26 in Eqs. 10 and 11, we get

$$
\begin{gathered}
A_{1}=-\frac{\left(m^{2}-k^{2}\right)}{2 \eta} B_{1}=\frac{i \omega}{2 \eta c} B_{1}, \\
A_{2}=\frac{2\left(v_{u}-v\right)}{\alpha_{0}} k^{2} B_{3},
\end{gathered}
$$

where

$$
v_{u}=v+\frac{\alpha_{0}^{2} T_{0}}{2 \mu\left(\rho C_{e}+\frac{\alpha_{0}^{2} T_{0}}{\mu(1-2 v)}\right)}
$$

The displacement components can now be written as:

$$
2 \mu u_{y}=-\int_{0}^{\infty}\left(B_{1} e^{-m z}+\left(B_{2}+B_{3}\left(2 v_{u}-2+k z\right)\right) e^{-k z}\right) k\left(\begin{array}{l}
\cos k y \\
-\sin k y
\end{array}\right) d k
$$

and

$$
2 \mu u_{z}=\int_{0}^{\infty}\left(m B_{1} e^{-m z}+\left(B_{2}+B_{3}\left(1-2 v_{u}+k z\right)\right) k e^{-k z}\right)\left(\begin{array}{c}
\sin k y \\
\cos k y
\end{array}\right) d k .
$$

The heat flux in $z$-direction is found as

$$
q_{z}=-\lambda_{0} \theta_{, z}=\lambda_{0} \int_{0}^{\infty}\left(m A_{1} e^{-m z}+k A_{2} e^{-k z}\right)\left(\begin{array}{c}
\sin k y \\
\cos k y
\end{array}\right) d k .
$$




\subsection{For elastic half space}

A homogeneous isotropic elastic medium can be characterized by the shear modulus $\left(\mu^{\prime}\right)$ and the Poisson's ratio $\left(v^{\prime}\right)$. The plane strain problem for an isotropic elastic medium can be solved in terms of the Airy stress function $\Phi$ such that

$$
\sigma_{y y}^{\prime}=\frac{\partial^{2} \Phi}{\partial z^{2}}, \quad \sigma_{z z}^{\prime}=\frac{\partial^{2} \Phi}{\partial y^{2}}, \quad \sigma_{y z}^{\prime}=-\frac{\partial^{2} \Phi}{\partial y \partial z}
$$

where $\Phi$ satisfies the biharmonic equation

$$
\nabla^{2} \nabla^{2} \Phi=0
$$

and $\sigma_{i j}^{\prime}$ are the components of stress tensor.

Let there be a line source parallel to the $x$-axis passing through the point $(0,0,-h)$ of the elastic half space $(z<0)$. The Airy stress function for a line source parallel to the $x$-axis passing through the point $(0,0,-h)$ in an unbounded isotropic elastic medium (Singh and Garg 1986), can be expressed in the form

$$
\Phi_{0}=\int_{0}^{\infty}\left(S_{1}+S_{2} k|z+h|\right) e^{-k|z+h|}\left(\begin{array}{c}
\sin k y \\
\cos k y
\end{array}\right) \frac{d k}{k} .
$$

The source coefficients $S_{1}$ and $S_{2}$ are independent of $k$.

The source coefficients for different types of sources, as given in Singh and Garg (1986), are given in Table 1. In this table, the upper sign is for $z>-h$, the lower sign is for $z<-h$ and

$$
\alpha^{\prime}=\frac{1}{2\left(1-v^{\prime}\right)} .
$$

The Airy stress function for the elastic half space can now be written as

$$
\Phi=\Phi_{0}+\int_{0}^{\infty}\left(C_{1}+C_{2} k z\right) e^{k z}\left(\begin{array}{c}
\sin k y \\
\cos k y
\end{array}\right) d k,
$$

where the unknowns $C_{1}, C_{2}$ are to be determined from the boundary conditions.

Using Eqs. 36, 38, and 39, the stresses are obtained as

$$
\begin{aligned}
& \sigma_{y y}^{\prime}=\int_{0}^{\infty}\left\{\left[S_{1}+S_{2}(-2+k|z+h|)\right] e^{-k|z+h|}+\left[C_{1}+C_{2}(k z+2)\right] k e^{k z}\right\}\left(\begin{array}{c}
\sin k y \\
\cos k y
\end{array}\right) k d k, \\
& \sigma_{z z}^{\prime}=-\int_{0}^{\infty}\left[\left(S_{1}+S_{2} k|z+h|\right) e^{-k|z+h|}+\left(C_{1}+C_{2} k z\right) k e^{k z}\right]\left(\begin{array}{c}
\sin k y \\
\cos k y
\end{array}\right) k d k,
\end{aligned}
$$


Table 1

Values of coefficients for different types of sources

\begin{tabular}{|c|c|c|c|}
\hline Source & $S_{1}$ & $S_{2}$ & $\begin{array}{c}\text { Upper or lower } \\
\text { solution }\end{array}$ \\
\hline Single couple $(y z)$ & $\mp \frac{F_{y z}}{2 \pi}$ & $\pm \alpha^{\prime} \frac{F_{y z}}{2 \pi}$ & upper \\
\hline Single couple $(z y)$ & $\pm \frac{F_{z y}}{2 \pi}$ & $\pm \alpha^{\prime} \frac{F_{z y}}{2 \pi}$ & upper \\
\hline $\begin{array}{l}\text { Double couple }(y z)+(z y) \\
F_{y z}=F_{z y}=D_{y z}\end{array}$ & 0 & $\pm \alpha^{\prime} \frac{D_{y z}}{\pi}$ & upper \\
\hline $\begin{array}{l}\text { Centre of rotation } \\
F_{y z}=F_{z y}=R_{y z}\end{array}$ & $\pm \frac{R_{y z}}{\pi}$ & 0 & upper \\
\hline Dipole (yy) & $\left(1-\alpha^{\prime}\right) \frac{F_{y y}}{2 \pi}$ & $-\alpha^{\prime} \frac{F_{y y}}{2 \pi}$ & lower \\
\hline Dipole $(z z)$ & $\left(1-\alpha^{\prime}\right) \frac{F_{z z}}{2 \pi}$ & $\alpha^{\prime} \frac{F_{z z}}{2 \pi}$ & lower \\
\hline $\begin{array}{l}\text { Centre of dilatation }(y y)+(z z) \\
\left(F_{y y}=F_{z z}=C_{0}\right)\end{array}$ & $\left(1-\alpha^{\prime}\right) \frac{C_{0}}{\pi}$ & 0 & lower \\
\hline $\begin{array}{l}\text { Double couple }(z z)-(y y) \\
\left(F_{y y}=F_{z z}=D_{y z}^{\prime}\right)\end{array}$ & 0 & $\alpha^{\prime} \frac{D_{y z}^{\prime}}{\pi}$ & lower \\
\hline
\end{tabular}

$$
\sigma_{y z}^{\prime}=\int_{0}^{\infty}\left\{ \pm\left[S_{1}-S_{2}(1-k|z+h|)\right] e^{-k|z+h|}-\left[C_{1}+C_{2}(1+k z)\right] k e^{k z}\right\}\left(\begin{array}{c}
\cos k y \\
-\sin k y
\end{array}\right) k d k
$$

The corresponding displacement components are

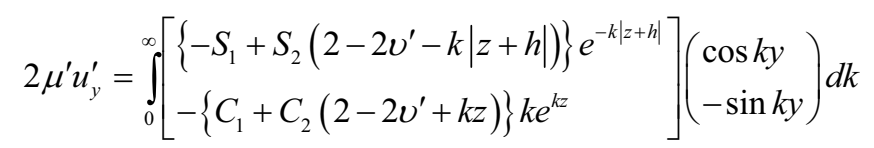

and

$$
2 \mu^{\prime} u_{z}^{\prime}=\int_{0}^{\infty}\left[\begin{array}{l} 
\pm\left\{S_{1}+S_{2}\left(1-2 v^{\prime}+k|z+h|\right)\right\} e^{-k|z+h|} \\
+\left\{-C_{1}+C_{2}\left(1-2 v^{\prime}-k z\right)\right\} k e^{k z}
\end{array}\right]\left(\begin{array}{l}
\sin k y \\
\cos k y
\end{array}\right) d k
$$

\section{BOUNDARY CONDITIONS}

The boundary conditions at the plane $z=0$ are

$$
\sigma_{y z}=\sigma_{y z}^{\prime}, \quad \sigma_{z z}=\sigma_{z z}^{\prime}, \quad u_{y}=u_{y}^{\prime}, \quad \text { and } u_{z}=u_{z}^{\prime} .
$$


Further, if the heat flux is not exchanged at the interface, then

$$
q_{z}=0, \quad \text { at } z=0 .
$$

It is noticed from Table 1 that the source coefficients $S_{1}$ and $S_{2}$ have different values according to $z>-h$ and $z<-h$. Let $S_{1}{ }^{\prime}$ and $S_{2}{ }^{\prime}$ be the values of $S_{1}$ and $S_{2}$ respectively, for $z>-h$. The boundary conditions 45 and 46 yield the system

$$
\begin{gathered}
m B_{1}+k B_{2}-k B_{3}+k C_{1}+k C_{2}=\left(S_{1}^{\prime}-S_{2}^{\prime}+S_{2}^{\prime} k h\right) e^{-k h} \\
k B_{1}+k B_{2}-k C_{1}=\left(S_{1}^{\prime}+S_{2}^{\prime} k h\right) e^{-k h} \\
k B_{1}+k B_{2}+2 k\left(v_{u}-1\right) B_{3}-\mu_{r} k C_{1}-2 \mu_{r} k\left(1-v^{\prime}\right) C_{2}=\mu_{r}\left(S_{1}^{\prime}+S_{2}^{\prime} k h-2 S_{2}^{\prime}+2 S_{2}^{\prime} v^{\prime}\right) e^{-k h}, \\
m B_{1}+k B_{2}+k\left(1-2 v_{u}\right) B_{3}+\mu_{r} k C_{1}-\mu_{r} k\left(1-2 v^{\prime}\right) C_{2}=\mu_{r}\left(S_{1}^{\prime}+S_{2}^{\prime} k h+S_{2}^{\prime}-2 S_{2}^{\prime} v^{\prime}\right) e^{-k h}, \\
m A_{1}+k A_{2}=0,
\end{gathered}
$$

where $\mu_{r}=\mu / \mu^{\prime}$.

On solving these equations, we have

$$
\begin{aligned}
& A_{1}=\frac{\Omega}{2 \eta} Q(k+m) S_{2}^{\prime} e^{-k h}, \quad A_{2}=-\frac{m}{k} A_{1}, \\
& B_{1}=\frac{\Omega}{k-m} Q S_{2}^{\prime} e^{-k h}, B_{2}=\left[-\frac{P_{2}}{k}\left(S_{1}^{\prime}-\frac{S_{2}^{\prime}}{2}+S_{2}^{\prime} k h\right)+\frac{Q S_{2}^{\prime}}{2 k}\left(1-\frac{k+m}{k-m} \Omega\right)\right] e^{-k h}, B_{3}=\frac{Q}{k} S_{2}^{\prime} e^{-k h}, \\
& C_{1}=\left[-\frac{P_{1}}{k}\left(S_{1}^{\prime}+S_{2}^{\prime} k h\right)+\frac{S_{2}^{\prime}}{2 k}\left(P_{2}+(1+\Omega) Q\right)\right] e^{-k h}, \quad C_{2}=\frac{P_{1}}{k}\left(2 S_{1}^{\prime}-S_{2}^{\prime}+2 S_{2}^{\prime} k h\right) e^{-k h},
\end{aligned}
$$

where

$$
\begin{aligned}
& P_{1}=\frac{1-\mu_{r}}{1+3 \mu_{r}-4 \mu_{r} v^{\prime}}, \quad P_{2}=P_{1}-1, \quad P_{3}=\frac{4 v_{u}-3-\mu_{r}}{1-\mu_{r}}, \\
& Q=\frac{P_{2}}{P_{1}\left(P_{3}+\Omega\right)}, \quad \Omega=\frac{k^{2} \gamma}{m(m+k)}, \quad \gamma=\frac{2\left(v-v_{u}\right)}{1-v} .
\end{aligned}
$$

Substituting the values of $A_{i}, B_{i}, C_{i}$ 's from Eqs. 52 in Eqs. 27-29, 33-34, 40-44, and 25, we get the integral expressions for the stress components and displacement components in Medium I and II, and the temperature difference in Medium I in terms of the source coefficients $S_{1}{ }^{\prime}$ and $S_{2}{ }^{\prime}$. 
These integrals can be solved numerically for arbitrary values of $\omega$. However, analytical solutions can be found for two particular cases:

Case (i) $\omega \rightarrow \infty$ which further implies that no net flow of heat takes place, i.e., the adiabatic condition.

Case (ii) $\omega \rightarrow 0$ which further implies that $\theta \rightarrow 0$, i.e., the isothermal condition.

\section{VERTICAL DIP SLIP DISLOCATION}

Following Maruyama (1966), the double couple $(y z)+(z y)$ is equivalent to a vertical dip slip dislocation so that its moment can be represented as

$$
D_{y z}=\mu^{\prime} b d s,
$$

where $b$ is the slip and $d s$ is the width of the line dislocation.

From Table 1, we have

$$
S_{1}^{\prime}=0, \quad S_{2}^{\prime}=\alpha^{\prime} \frac{D_{y z}}{\pi}=\frac{D_{y z}}{2 \pi\left(1-v^{\prime}\right)},
$$

with the stipulation that in the representation of integrals, the upper sign is to be selected.

The results for the two limiting cases, $\omega \rightarrow \infty$ and $\omega \rightarrow 0$, are obtained in analytical form as follows:

\section{Case (i) Adiabatic case}

$$
\begin{aligned}
& U=\alpha^{\prime} \frac{D_{y z}}{\pi}\left[\frac{1}{2}\left(P_{2}+Q_{2}\right) \tan ^{-1}\left(\frac{y}{z+h}\right)+\left(-P_{2} h+Q_{2} z\right) \frac{y}{R_{1}^{2}}\right] \\
& \sigma_{y y}=\frac{\alpha^{\prime} D_{y z}}{\pi} \frac{y}{R_{1}^{4}}\left[P_{2}(z+3 h)-Q_{2}(5 z+3 h)+8\left(Q_{2} z-P_{2} h\right) \frac{(z+h)^{2}}{R_{1}^{2}}\right] \\
& \sigma_{y z}=\frac{\alpha^{\prime} D_{y z}}{\pi} \frac{1}{R_{1}^{2}}\left[\frac{1}{2}\left(Q_{2}-P_{2}\right)+\left\{P_{2}(z+7 h)-Q_{2}(7 z+h)\right\} \frac{(z+h)}{R_{1}^{2}}+\frac{8\left(Q_{2} z-P_{2} h\right)(z+h)^{3}}{R_{1}^{4}}\right], \\
& \sigma_{z z}=-\frac{\alpha^{\prime} D_{y z}}{\pi} \frac{y}{R_{1}^{4}}\left[P_{2}(z+3 h)-Q_{2}(z-h)+8\left(Q_{2} z-P_{2} h\right) \frac{(z+h)^{2}}{R_{1}^{2}}\right] \\
& \varepsilon_{y y}=\frac{\alpha^{\prime} D_{y z}}{2 \mu \pi} \frac{y}{R_{1}^{4}}\left[P_{2}(z+3 h)-Q_{2}\left(5 z+3 h+\left(\frac{\alpha_{0} \gamma}{\eta}-4 v\right)(z+h)\right)+8\left(Q_{2} z-P_{2} h\right) \frac{(z+h)^{2}}{R_{1}^{2}}\right],
\end{aligned}
$$




$$
\begin{aligned}
& \varepsilon_{y z}=\frac{\alpha^{\prime} D_{y z}}{2 \mu \pi} \frac{1}{R_{1}^{2}}\left[\frac{1}{2}\left(Q_{2}-P_{2}\right)+\left\{P_{2}(z+7 h)-Q_{2}(7 z+h)\right\} \frac{(z+h)}{R_{1}^{2}}+\frac{8\left(Q_{2} z-P_{2} h\right)(z+h)^{3}}{R_{1}^{4}}\right], \\
& \varepsilon_{z z}=-\frac{\alpha^{\prime} D_{y z}}{2 \mu \pi} \frac{y}{R_{1}^{4}}\left[P_{2}(z+3 h)-Q_{2}\left((z-h)+\left(4 v-\frac{\alpha_{0} \gamma}{\eta}\right)(z+h)\right)+8\left(Q_{2} z-P_{2} h\right) \frac{(z+h)^{2}}{R_{1}^{2}}\right], \\
& u_{y}=\frac{\alpha^{\prime} D_{y z}}{2 \mu \pi} \frac{1}{R_{1}^{2}}\left[-\frac{1}{2}\left[P_{2}(z+3 h)+Q_{2}(h-z)-4 Q_{4}(z+h)\right]-2\left(Q_{2} z-P_{2} h\right) \frac{(z+h)^{2}}{R_{1}^{2}}\right], \\
& u_{z}=\frac{\alpha^{\prime} D_{y z}}{2 \mu \pi} \frac{y}{R_{1}^{2}}\left[\frac{1}{2}\left(P_{2}-Q_{2}+4 Q_{4}\right)+2\left(Q_{2} z-P_{2} h\right) \frac{(z+h)}{R_{1}^{2}}\right] \\
& \theta=-\frac{\alpha^{\prime} D_{y z}}{\pi}\left(\frac{\gamma}{\eta}\right) Q_{2} \frac{y(z+h)}{R_{1}^{4}}
\end{aligned}
$$

where $R_{1}^{2}=y^{2}+(z+h)^{2}, Q_{2}=P_{2} / P_{1} P_{3}$, and $Q_{4}=Q_{2}\left(1-v_{u}\right)$.

For the above limiting case, the solutions for the elastic half space are obtained as follows:

$$
\begin{aligned}
& \Phi=\frac{\alpha^{\prime} D_{y z}}{\pi}\left[y(z+h) \frac{1}{R_{1}^{2}}+\frac{1}{2}\left(P_{2}+Q_{2}\right) \tan ^{-1}\left(\frac{y}{h-z}\right)-P_{1} \frac{y(z+h)}{R_{2}^{2}}+4 P_{1} h \frac{y z(h-z)}{R_{2}^{4}}\right], \\
& \sigma_{y y}^{\prime}=\frac{\alpha^{\prime} D_{y z} y}{\pi}\left[2\left(-3+4 \frac{(z+h)^{2}}{R_{1}^{2}}\right) \frac{(z+h)}{R_{1}^{4}}+\left(P_{2}+Q_{2}\right) \frac{(h-z)}{R_{2}^{4}}-2 P_{1}(5 h-3 z) \frac{1}{R_{2}^{4}}\right. \\
& \left.+8 P_{1}(h-z)\left(z^{2}+3 h^{2}-10 h z\right) \frac{1}{R_{2}^{6}}+96 P_{1} h z \frac{(h-z)^{3}}{R_{2}^{8}}\right], \\
& \sigma_{z z}^{\prime}=\frac{\alpha^{\prime} D_{y z} y}{\pi}\left[2\left(1-4 \frac{(z+h)^{2}}{R_{1}^{2}}\right) \frac{(z+h)}{R_{1}^{4}}-\left(2 P_{1}(z+h)+\left(P_{2}+Q_{2}\right)(h-z)\right.\right. \\
& \left.\left.-8 P_{1}(h-z)\left(h^{2}-z^{2}+6 h z\right) \frac{1}{R_{2}^{2}}+96 P_{1} h z \frac{(h-z)^{3}}{R_{2}^{4}}\right) \frac{1}{R_{2}^{4}}\right], \\
& \sigma_{y z}^{\prime}=\frac{\alpha^{\prime} D_{y z}}{\pi}\left[\left(1-8\left(\frac{z+h}{R_{1}}\right)^{2}+8\left(\frac{z+h}{R_{1}}\right)^{4}\right) \frac{1}{R_{1}^{2}}+\left(\frac{1}{2}\left(P_{2}+Q_{2}-2 P_{1}\right)+\left(8 P_{1}-P_{2}-Q_{2}\right) \frac{(h-z)^{2}}{R_{2}^{2}}\right.\right. \\
& \left.\left.-12 P_{1} h z \frac{1}{R_{2}^{2}}-8 P_{1} \frac{(h-z)^{4}}{R_{2}^{4}}\right) \frac{1}{R_{2}^{2}}+96 P_{1} h z \frac{(h-z)^{2}}{R_{2}^{6}}\left(1-\left(\frac{h-z}{R_{2}}\right)^{2}\right)\right] \text {, }
\end{aligned}
$$




$$
\begin{aligned}
& \varepsilon_{y y}^{\prime}=\frac{\alpha^{\prime} D_{y z} y}{2 \mu^{\prime} \pi}\left[2\left(-3+2 v+4 \frac{(z+h)^{2}}{R_{1}^{2}}\right) \frac{(z+h)}{R_{1}^{4}}+\left(P_{2}+Q_{2}\right) \frac{(h-z)}{R_{2}^{4}}\right.-2 P_{1}(5 h-3 z-v(6 \mathrm{~h}-2 z)) \frac{1}{R_{2}^{4}} \\
&\left.+8 P_{1}(h-z)\left(z^{2}+3 h^{2}-10 h z-4 v h(\mathrm{~h}-\mathrm{z})\right) \frac{1}{R_{2}^{6}}+96 P_{1} h z \frac{(h-z)^{3}}{R_{2}^{8}}\right], \quad(70) \\
& \varepsilon_{z z}^{\prime}=\frac{\alpha^{\prime} D_{y z} y}{2 \mu^{\prime} \pi}\left[2\left(1+2 v-4 \frac{(z+h)^{2}}{R_{1}^{2}}\right) \frac{(z+h)}{R_{1}^{4}}-\left\{2 P_{1}((z+h)-v(6 h-2 z))+\left(P_{2}+Q_{2}\right)(h-z)\right.\right.\left.\left.-8 P_{1}(h-z)\left(h^{2}-z^{2}+6 h z-4 v(h-z)\right) \frac{1}{R_{2}^{2}}+96 P_{1} h z \frac{(h-z)^{3}}{R_{2}^{4}}\right\} \frac{1}{R_{2}^{4}}\right], \\
& \varepsilon_{y z}^{\prime}=\frac{\alpha^{\prime} D_{y z}}{2 \mu^{\prime} \pi}\left[\left(1-8\left(\frac{z+h}{R_{1}}\right)^{2}+8\left(\frac{z+h}{R_{1}}\right)^{4}\right) \frac{1}{R_{1}^{2}}+\left(\frac{1}{2}\left(P_{2}+Q_{2}-2 P_{1}\right)+\left(8 P_{1}-P_{2}-Q_{2}\right) \frac{(h-z)^{2}}{R_{2}^{2}}\right.\right. \\
&\left.\left.-12 P_{1} h z \frac{1}{R_{2}^{2}}-8 P_{1} \frac{(h-z)^{4}}{R_{2}^{4}}\right) \frac{1}{R_{2}^{2}}+96 P_{1} h z \frac{(h-z)^{2}}{R_{2}^{6}}\left(1-\left(\frac{h-z}{R_{2}}\right)^{2}\right)\right],
\end{aligned}
$$$$
u_{y}^{\prime}=\frac{\alpha^{\prime} D_{y z}}{2 \mu^{\prime} \pi}\left[\left(3-2 v^{\prime}-2 \frac{(z+h)^{2}}{R_{1}^{2}}\right) \frac{(z+h)}{R_{1}^{2}}-\left(\frac{1}{2}\left(P_{2}+Q_{2}\right)(h-z)+P_{1}(h+z)\right.\right.
$$$$
\left.+2\left(1-v^{\prime}\right) P_{1}(z-3 h)\right) \frac{1}{R_{2}^{2}}
$$

$$
\begin{aligned}
&\left.+2 P_{1}\left(h^{2}-z^{2}+6 h z-4\left(1-v^{\prime}\right) h(h-z)\right) \frac{(h-z)}{R_{2}^{4}}-16 P_{1} h z \frac{(h-z)^{3}}{R_{2}^{6}}\right], \\
& u_{z}^{\prime}=\frac{\alpha^{\prime} D_{y z} y}{2 \mu^{\prime} \pi}\left[\left(\left(1-2 v^{\prime}\right)+2 \frac{(z+h)^{2}}{R_{1}^{2}}\right) \frac{1}{R_{1}^{2}}-\left(\frac{1}{2}\left(P_{2}+Q_{2}\right)+P_{1}\left(1-2 v^{\prime}\right)\right) \frac{1}{R_{2}^{2}}\right. \\
&\left.+2 P_{1}\left(3 h^{2}-z^{2}-4 v^{\prime} h(h-z)\right) \frac{1}{R_{2}^{4}}-16 P_{1} h z \frac{(h-z)^{2}}{R_{2}^{6}}\right],
\end{aligned}
$$

where $R_{2}^{2}=y^{2}+(z-h)^{2}$.

\section{Case (ii) Isothermal case}

To obtain the results for this particular case, we define

$$
P_{4}=\frac{4 v-3-\mu_{r}}{1-\mu_{r}} \quad Q_{1}=\frac{P_{2}}{P_{1} P_{4}} \quad \text { and } \quad Q_{3}=(1-v) Q_{1} .
$$


The expressions for stress components and displacement components for the present case of isothermal are similar to that for the Case (i) if we replace $Q_{2}$ by $Q_{1}$ and $Q_{4}$ by $Q_{3}$. These results coincide with the corresponding expressions for a source in an elastic half space in welded contact with another elastic half space (Singh et al. 1992).

\section{NUMERICAL RESULTS AND DISCUSSION}

Equations 57-64 and 67-74 can be used for computing the stresses, strain and displacements in the two half spaces under adiabatic (thermal equilibrium) conditions. The temperature difference is given by Eq. 65. To obtain the results for the stresses, strain, and displacements under isothermal conditions, $Q_{2}$ and $Q_{4}$ are changed to $Q_{1}$ and $Q_{3}$ respectively, in Eqs. 57-64 and 67-74. Following Aki and Richards (1980) and Ahrens (1995), the parameters for thermoelastic medium (Pyrope rich garnet) are taken as

$$
\begin{aligned}
& \mu=8.51514 \times(10)^{10} \mathrm{~kg} \mathrm{~m}^{-1} \mathrm{~s}^{-2}, \lambda=11.4508 \times(10)^{10} \mathrm{~kg} \mathrm{~m}^{-1} \mathrm{~s}^{-2}, \alpha_{t}=3.11 \times(10)^{-5} \mathrm{~K}^{-1}, \\
& \rho=3620 \mathrm{~kg} \mathrm{~m}^{-3}, T_{0}=1000 \mathrm{~K}, C_{e}=1076 \mathrm{~m}^{2} \mathrm{~K}^{-1} \mathrm{~s}^{-2}, \quad \beta=(3 \lambda+2 \mu) \alpha_{t} .
\end{aligned}
$$

For the elastic half space, the Poisson ratio $\left(v^{\prime}\right)$ is taken as 0.25 . To make the quantities dimensionless, the followings are defined

$$
\begin{aligned}
& Y=\frac{y}{h}, \quad Z=\frac{z}{h}, \quad T=\frac{2 c t}{h^{2}}, \quad U_{i}^{\prime}=\frac{h}{b d s} u_{i}, \\
& \Theta=\frac{h^{2}}{\mu^{\prime} b d s} \theta, \quad \Sigma_{i j}=\frac{h^{2}}{\mu^{\prime} b d s} \sigma_{i j}, \quad \Sigma_{i j}^{\prime}=\frac{h^{2}}{\mu^{\prime} b d s} \sigma_{i j}^{\prime} .
\end{aligned}
$$

The displacements, strain and stresses generated by a vertical dip-slip dislocation located at a point $(0,0,-h)$ of the elastic half space are computed.

For $z=0$, the displacement components for both the limiting cases, adiabatic and isothermal, are computed and shown in Fig. 2 for $\mu_{r}=0.5$. Here $\mu_{r}$ is the ratio of rigidity of thermoelastic and elastic half spaces. For $\mu_{r}=2$, the displacement components are compared in Fig. 3. It is noticed that the difference between adiabatic and isothermal deformations is more significant when the thermoelastic half space is more rigid than the elastic half space. The more rigid the elastic half space, the larger the horizontal and vertical displacements are.

Assuming that $\mu_{r}=2$, the variation of the dimensionless stresses $\left(\Sigma_{y y}\right.$, $\Sigma_{z z}$, and $\Sigma_{y z}$ ) with dimensionless horizontal distance $(Y)$ from the fault is shown in Figs. 4a, 5a, and 6a for $z=0$ and that for $z=h$ is shown in Figs. $4 \mathrm{~b}, 5 \mathrm{~b}$, and $6 \mathrm{~b}$. At the interface $z=0$, the stresses $\Sigma_{z z}$ and $\Sigma_{y z}$ first decrease with $Y$ and then increase steadily when horizontal distance is a little 
(a)

(b)
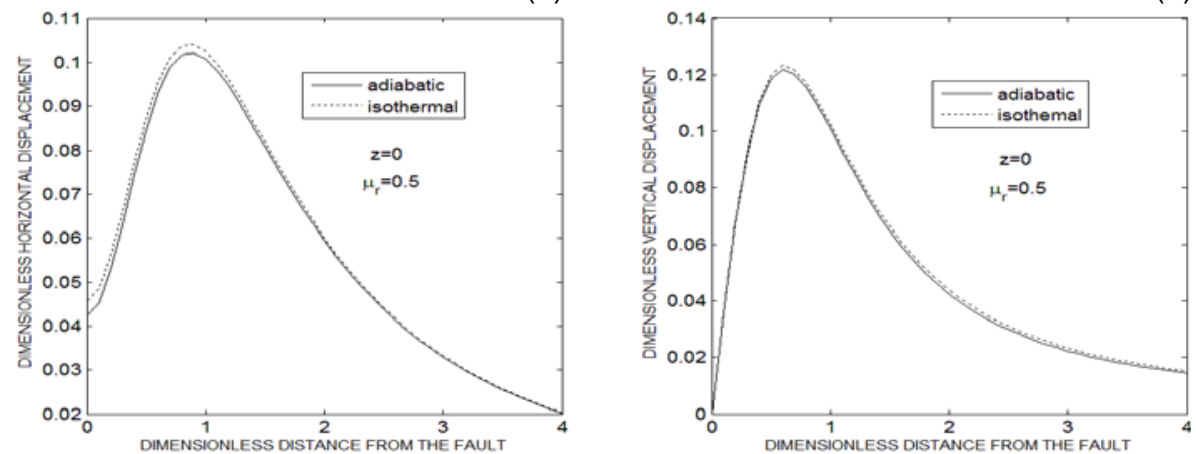

Fig. 2. Variation of horizontal and vertical displacements at the interface $z=0$ with horizontal distance from the fault for $\mu_{r}=0.5$.

(a)

(b)
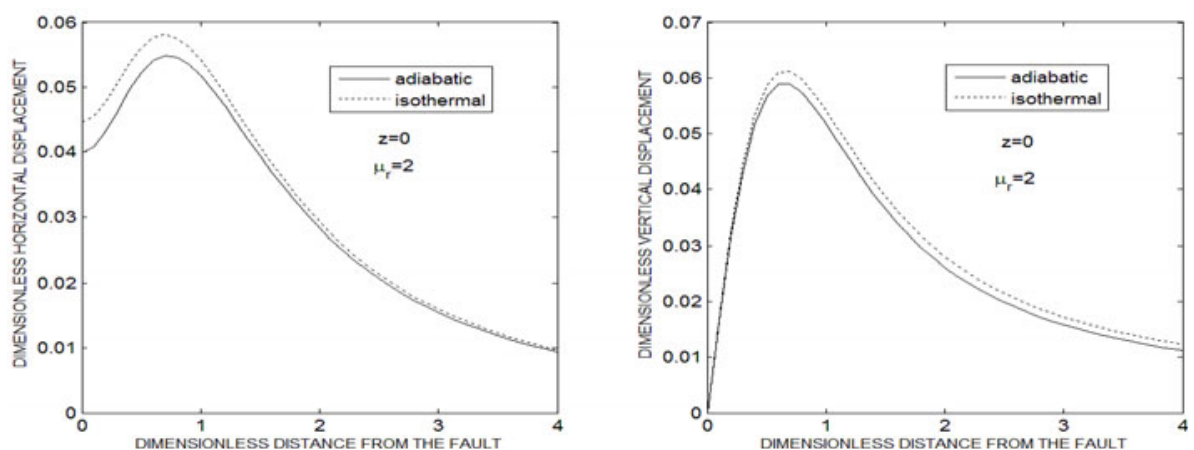

Fig. 3. Variation of horizontal and vertical displacements at the interface $z=0$ with horizontal distance from the fault for $\mu_{r}=2$.

(a)

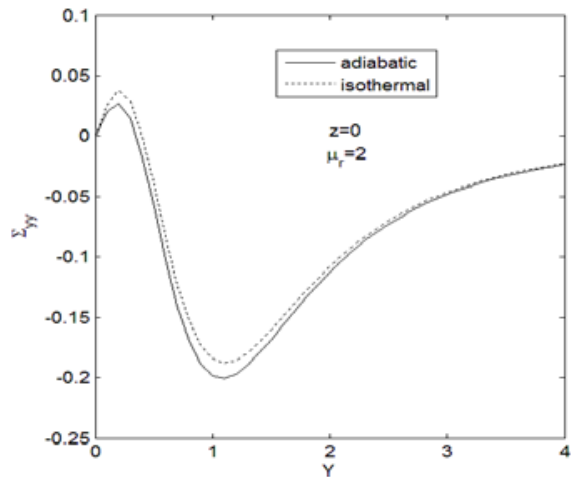

(b)

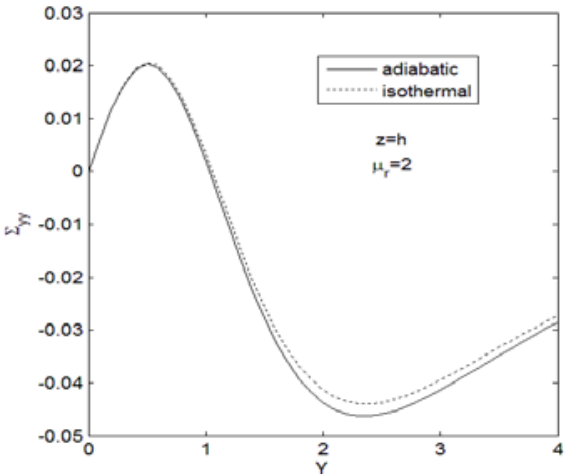

Fig. 4. Variation in dimensionless stress $\Sigma_{y y}$ in the thermoelastic half space with horizontal distance from the fault $z=0$ (a), and $z=h(\mathrm{~b})$. 
(a)

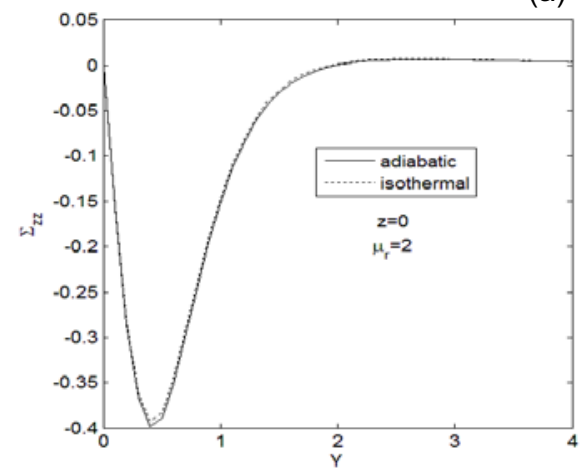

(b)

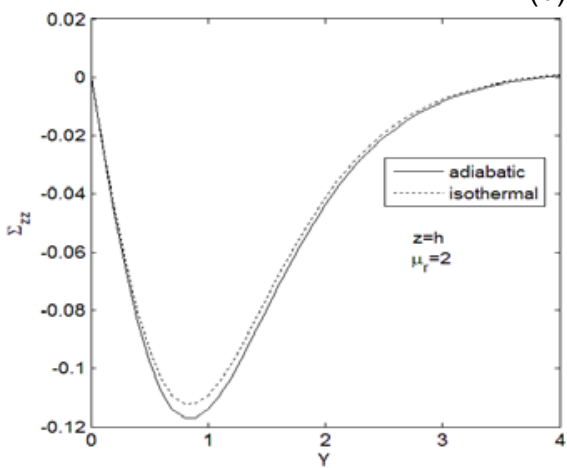

Fig. 5. Variation in dimensionless stress $\Sigma_{z z}$ in the thermoelastic half space with horizontal distance from the fault $z=0$ (a), and $z=h(\mathrm{~b})$.

(a)

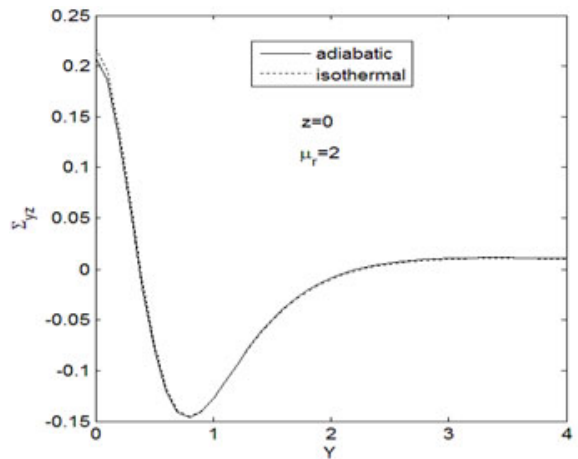

(b)

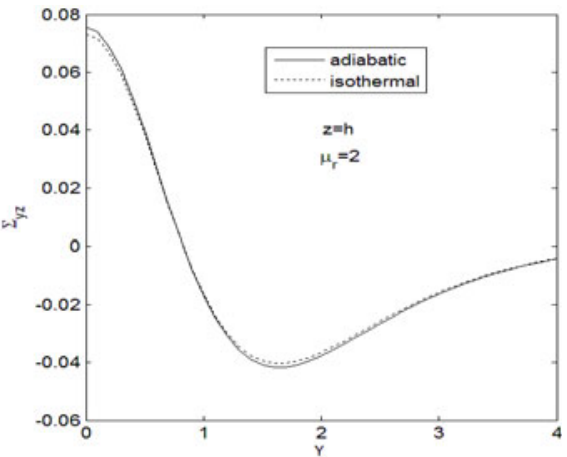

Fig. 6. Variation in dimensionless stress $\Sigma_{y z}$ in the thermoelastic half space with horizontal distance from the fault $z=0$ (a), and $z=h(\mathrm{~b})$.

less than the depth of the source. As the horizontal distance increases further, i.e., more than twice of the source depth, $\Sigma_{z z}$ and $\Sigma_{y z}$ approach to zero. However, $\Sigma_{y y}$ first increases then decreases and then again increases with $Y$. The variation pattern of $\Sigma_{y y}, \Sigma_{z z}$, and $\Sigma_{y z}$ gets smoother for $z=h$, as evident from Figs. 4b-6b. In Figure 7, depth profiles of the stresses $\Sigma_{y y}, \Sigma_{z z}$, and $\Sigma_{y z}$ are exhibited for $y=h, \mu_{r}=2$. The stress $\Sigma_{y y}$ has a maximum value near the plane of source, i.e., $z=-h$. As it goes away from the plane of source towards the interface, it firstly decreases and then increases. It experiences a discontinuity at the interface. Below the plane $z=h$ and above the plane $z=-3 h$, the stress $\Sigma_{\mathrm{yy}}$ becomes stable and approaches to zero. In the case of $\Sigma_{y z}$ and $\Sigma_{z z}$, the stresses are continuous at the interface, as expected from the boundary conditions. As we move away from the interface in the elastic medium, $\Sigma_{z z}$ increases up to a little before the source plane $z=-h$, decreases and 
(a)

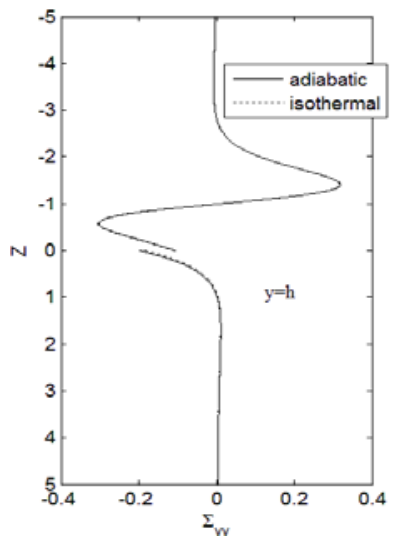

(b)

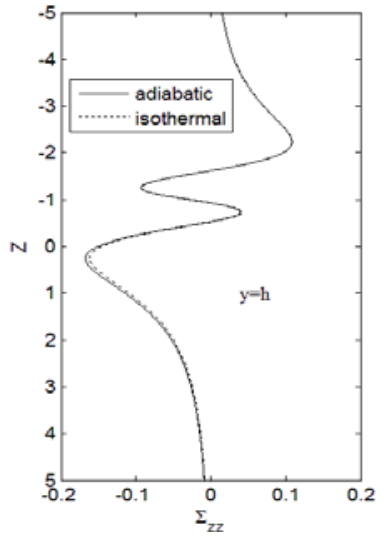

(c)

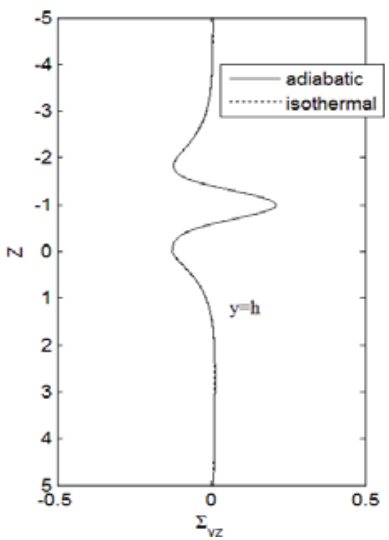

Fig. 7. Variation in dimensionless stresses $\Sigma_{y y}$ (a), $\Sigma_{z z}$ (b), and $\Sigma_{y z}$ (c) with distance from the interface for $y=h$.

increases rapidly up to $z=-2 h$, then smoothly decreases and approaches to zero. Similarly, in the thermoelastic half space, it increases smoothly and approaches to zero. The stress $\Sigma_{y z}$ is greatest on the plane of source, decreases up to a distance $h$ and then increases and approach to zero which is a physically plausible situation also. It is symmetric about the plane of source. No major difference in the variation of the stresses for the two limiting cases is noticed.

From the Eqs. 25, 27-29, 33-34, and 40-44, we get the integral expressions for the temperature, displacements, and stresses at any point in each of the two half spaces caused by a vertical dip slip dislocation located at the point $(0,0,-h)$ of the elastic half space in $k-\omega$ domain. On replacing $(-i \omega)$ by $s$, we get the solutions in Fourier-Laplace transform domain, where $s$ is the Laplace transforming variable. Two integrations are required to be performed to get the solution in the space time domain. Schapery (1962) proposed a very simple and efficient approximate formula for finding Laplace inversion numerically. Accordingly, it can be written as

$$
\theta(t) \approx[s \bar{\theta}(s)]_{s=1 /(2 t)},
$$

where $\bar{\theta}(s)$ is the Laplace transform of $\theta(t)$.

Inverse Fourier transform is computed numerically. Due to exponential decay, the integrands decrease very rapidly with $k$.

Figure 8 demonstrates the variation of temperature with time at different depths. It is noticed that the deviation is significant near the interface and the temperature difference approaches to zero as time increases at all depth. 


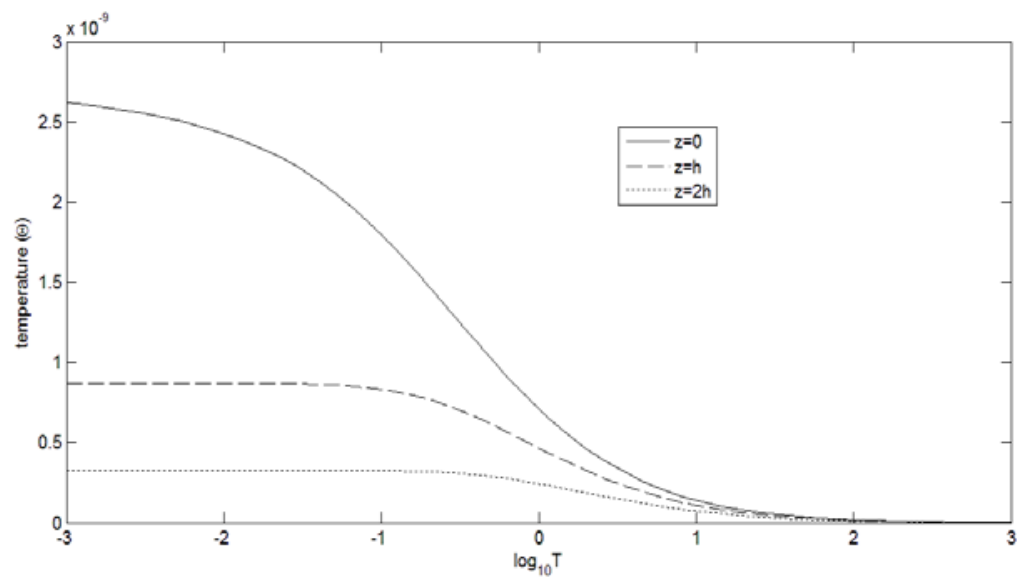

Fig. 8. Diffusion of the temperature difference $\Theta$ with $T$ for $y=h$ and $z=0, h, 2 h$.

(a)

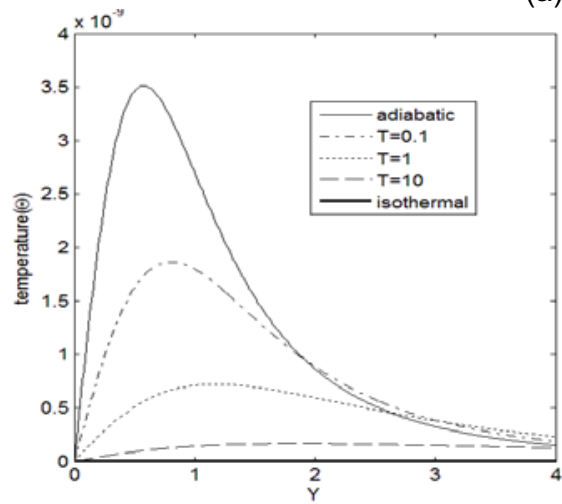

(b)

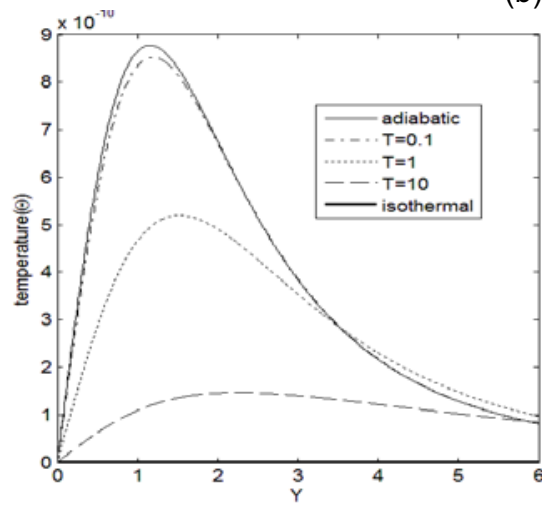

Fig. 9. Variation of the temperature difference $\Theta$ with the horizontal distance $Y$ from the fault for $y=h$ at five times $T=0,0.1,1,10, \infty$ for $z=0$ (a), and $z=h(\mathrm{~b})$.

Temperature difference's variation with $Y$ for different times $T=0$ (adiabatic), 0.1, 1, 10, $\infty$ (isothermal), for $z=0$ and $z=h$ is shown in Fig. 9. As expected, the temperature difference is found zero in the isothermal state. The point of maxima of the temperature difference moves away from the source with time. The temperature difference at different times is more significant on the interface. For all the times, the curves tend to merge for large $Y$. As $T$ increases, maximum value of temperature difference decreases and maxima travels rightwards with $Y$.

Figure 10 depicts the depth profile of temperature difference at different times: $T=0$ (adiabatic), $0.1,1,10, \infty$ (isothermal). Temperature difference is greatest on the interface and strongly depends on time. As the distance from the interface increases, the temperature difference diffuses rapidly. 


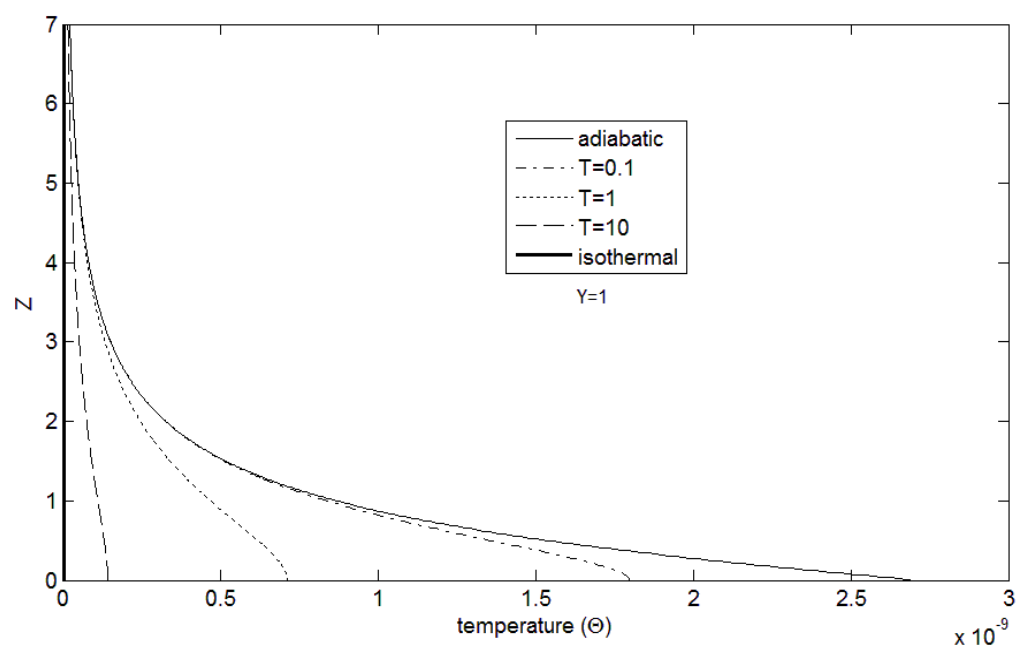

Fig. 10. Variation of the temperature difference $\Theta$ with the distance from the interface for $y=h$ at $T=0,0.1,1,10, \infty$.

Figures 11-15 exhibit time history of dimensionless displacements and stresses for $y=h$ : (a) $z=0$, and (b) $z=2 h$. It is noticed that, at the interface, horizontal displacement and $\Sigma_{y z}$ decrease slowly with $T$ and then increase, but the vertical displacement and stresses $\Sigma_{y y}$ and $\Sigma_{z z}$ increase with time. At $z=2 h$, the horizontal displacement and $\Sigma_{z z}$ increase with time but the vertical displacement, stresses $\Sigma_{y y}$ and $\Sigma_{y z}$ firstly decrease then increase. It is noticed that as the distance from the interface increases, the point of minima of the displacements and stresses move rightward along the time. Also, the variation in displacements and stresses is significant in the range $T=0.01$ to $T=100$.

The stress $\Sigma_{y y}$ 's variation with temperature and with horizontal distance from the fault is shown in Fig. 16. Similarly, the stresses profiles of $\Sigma_{\mathrm{zz}}$ and $\Sigma_{y z}$ are shown in the Figs. 17 and 18. It can be concluded from these graphs that temperature distribution does not have a major role in the determination of stresses in the thermoelastic half space except in the vicinity of the fault plane. In Fig. 19, the stress profiles with temperature and distance from the interface are depicted. These graphs also confirm the observations about stress variation with $Z$ made earlier.

Deformation in elastic medium is presented in Figs. 20-23.Variation of horizontal and vertical displacements at $z=-1.5 h$ with horizontal distance from the fault are presented in Fig. 20 and that at $z=-5 h$ are presented in Fig. 21. Horizontal displacement first decreases and then increases and approaches to zero. Vertical displacement first increases, then deceases and 
(a)
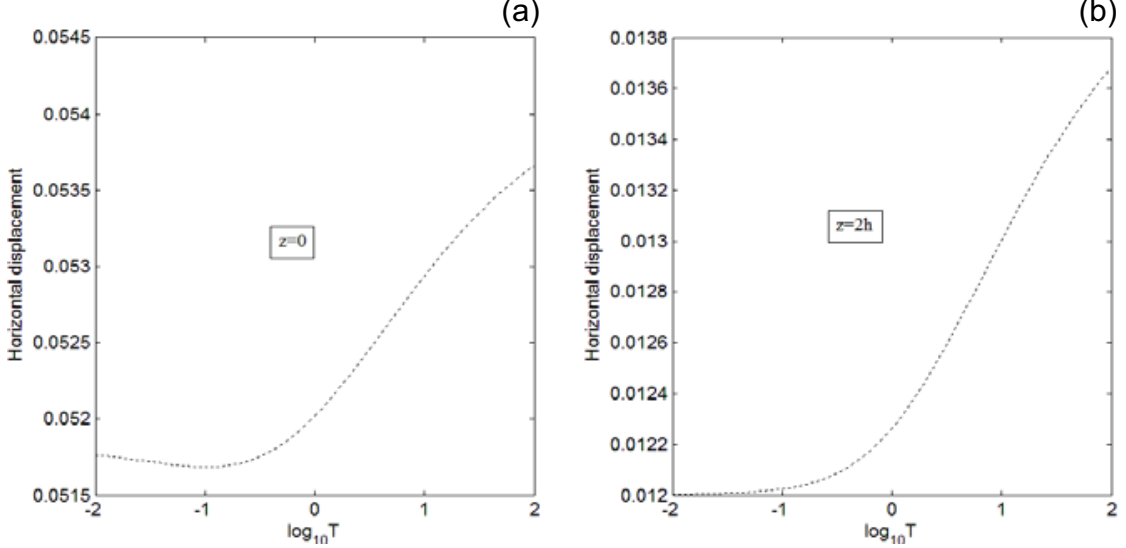

Fig. 11. Variation of horizontal displacement with time $T:$ (a) $z=0$, and (b) $z=2 h$.

(a)

(b)
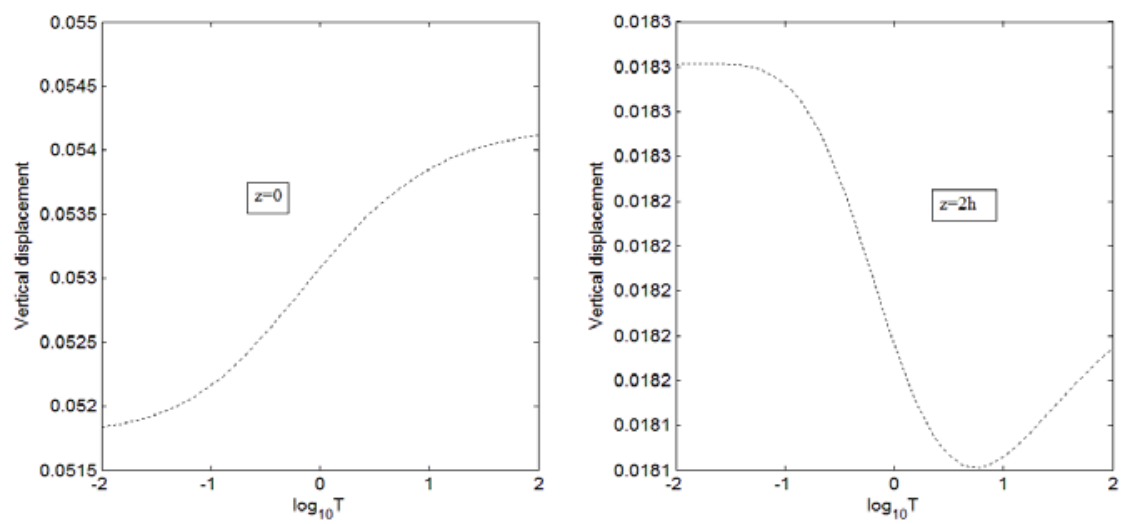

Fig. 12. Variation of vertical displacement with time $T:$ (a) $z=0$, and (b) $z=2 h$.

(a)

(b)
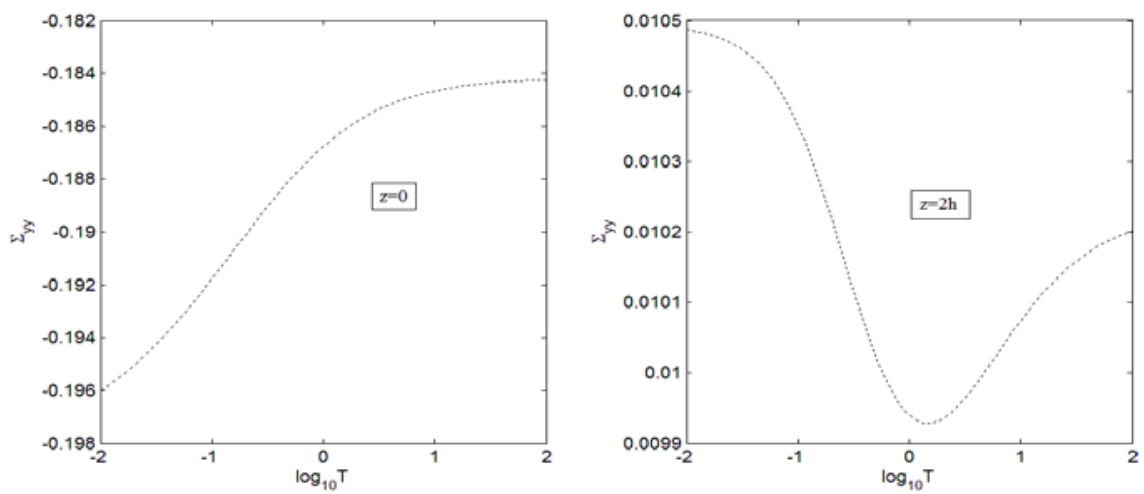

Fig. 13. Variation of stress $\Sigma_{y y}$ with time $T:$ (a) $z=0$, and (b) $z=2 h$. 
(a)

(b)
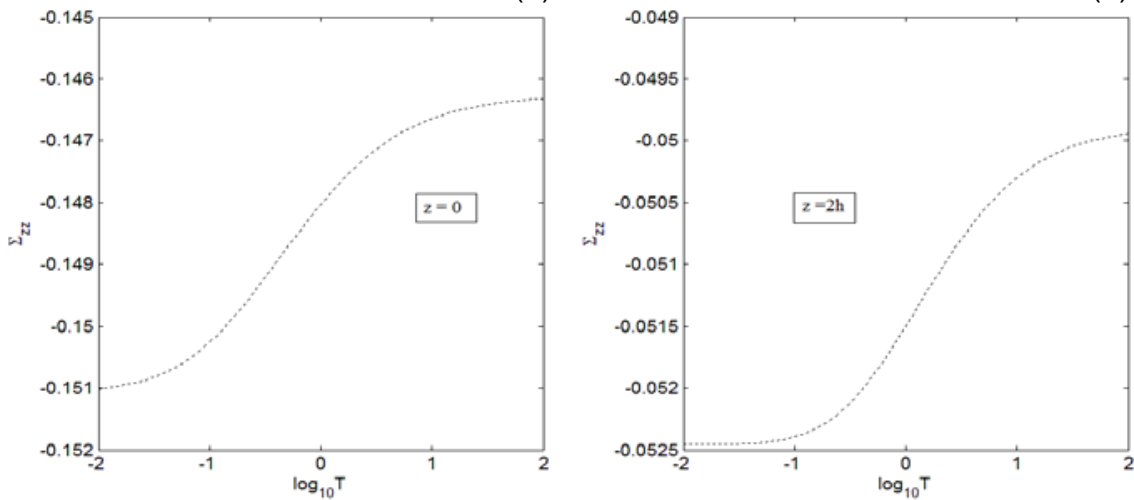

Fig. 14. Variation of stress $\Sigma_{z z}$ with time $T$ : (a) $z=0$, and (b) $z=2 h$.

(a)
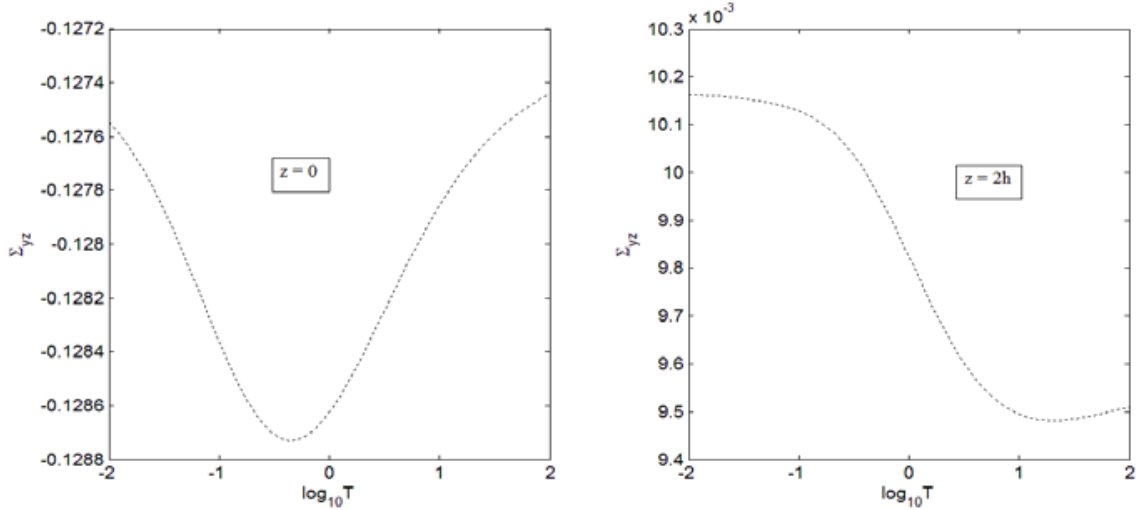

Fig. 15. Variation of stress $\Sigma_{y z}$ with time $T$ : (a) $z=0$, and (b) $z=2 h$.

(a)

(b)
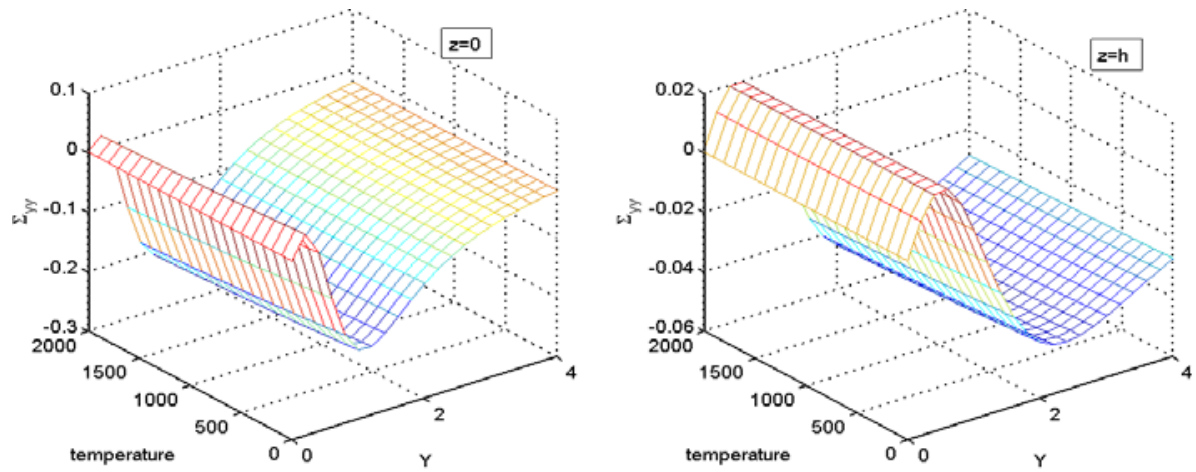

Fig. 16. Variation of stresses $\Sigma_{y y}$ with temperature and distance from fault $z=0$ (a), and $z=h(\mathrm{~b})$. 
(a)

(b)
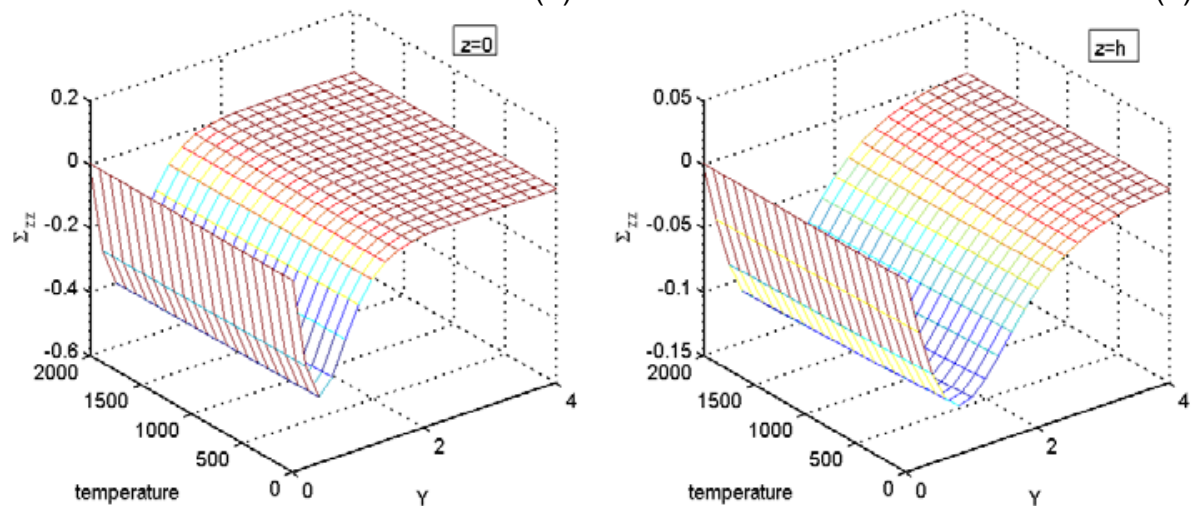

Fig. 17. Variation of stresses $\Sigma_{z z}$ with temperature and distance from fault $z=0$ (a), and $z=h(\mathrm{~b})$.

(a)

(b)
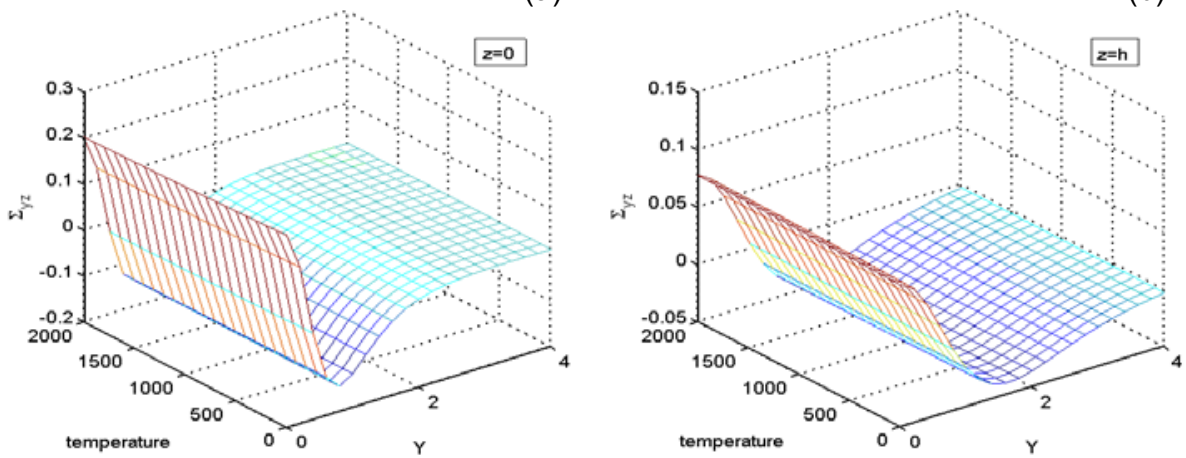

Fig. 18. Variation of stresses $\Sigma_{y z}$ with temperature and distance from fault $z=0$ (a), and $z=h(\mathrm{~b})$.

(a)

(b)

(c)
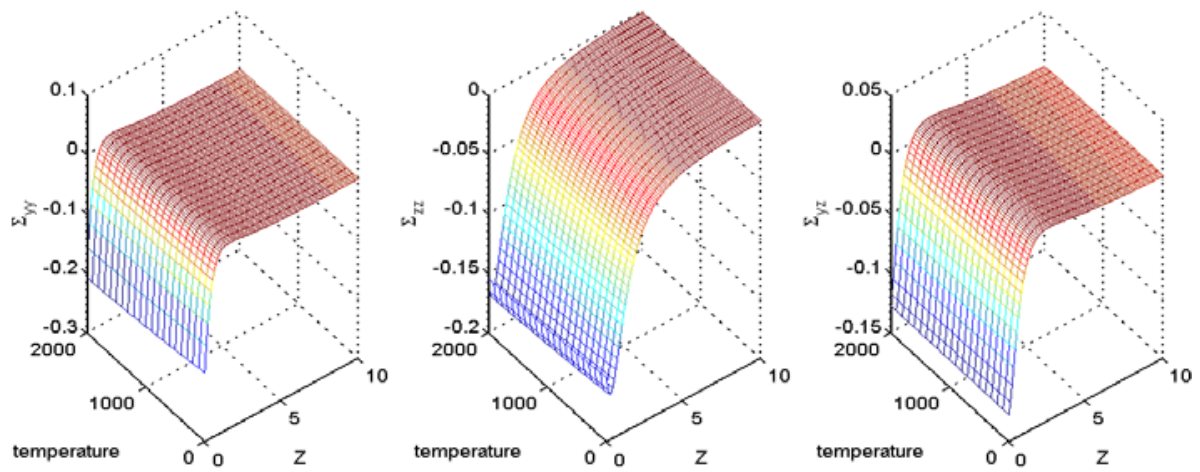

Fig. 19. Variation of stresses $\Sigma_{y y}$ (a), $\Sigma_{z z}$ (b), and $\Sigma_{y z}$ (c) with temperature and distance from interface for $y=h$. 
(a)

(b)
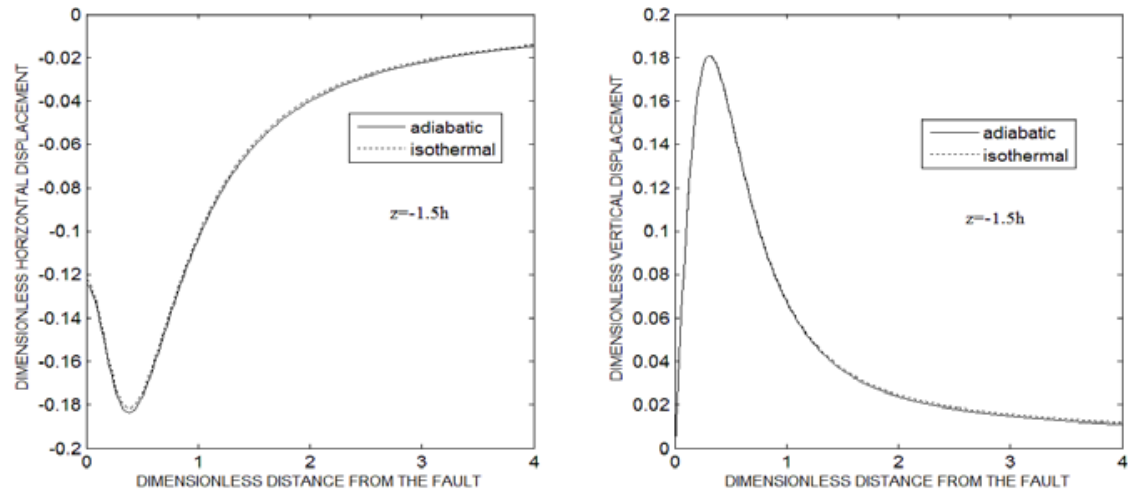

Fig. 20. Variation of horizontal and vertical displacements at $z=-1.5 h$ with horizontal distance from the fault.

(a)

(b)
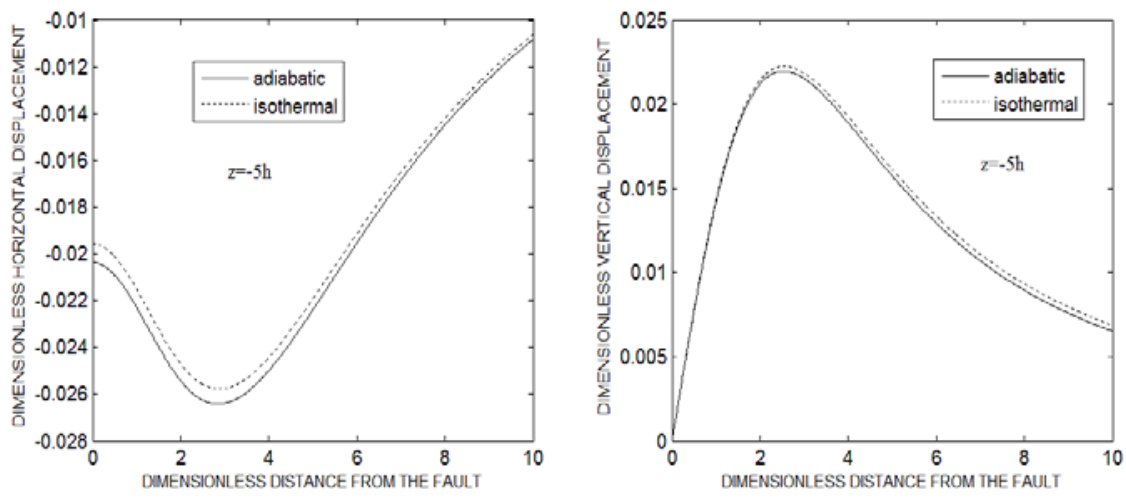

Fig. 21. Variation of horizontal and vertical displacements at $z=-5 h$ with horizontal distance from the fault.

approaches to zero. It is noticed that difference in the two extreme cases (adiabatic and isothermal) is more significant at $z=-5 h$ than $z=-1.5 h$ but the displacements are more significant near the plane of source (i.e., $z=-1.5 h)$.

Variation of the stresses $\Sigma_{y y}^{\prime}, \Sigma_{z z}^{\prime}, \Sigma_{y z}^{\prime}$ at $z=-1.5 h$ with horizontal distance from the fault are presented in Fig. 22a, b and that at $z=-5 h$ are presented in Fig. 23a, b. The difference in the limiting cases is significant at far distance from the source. There is a sharp stress drop in elastic half space, i.e., the region containing the fault in comparison to the other half space. At a sufficient distance from the source, the shear displacement increases steadily and the longitudinal displacement decreases steadily. 
(a)

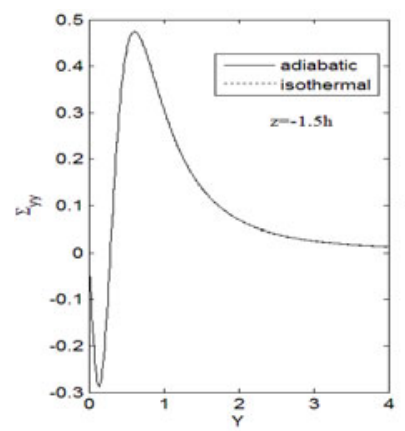

(b)

(c)

Fig. 22. Variation of stresses $\Sigma_{y y}^{\prime}$ (a), $\Sigma_{z z}^{\prime}$ (b), and $\Sigma_{y z}^{\prime}$ (c) at $z=-1.5 h$ with horizontal distance from the fault.

(a)

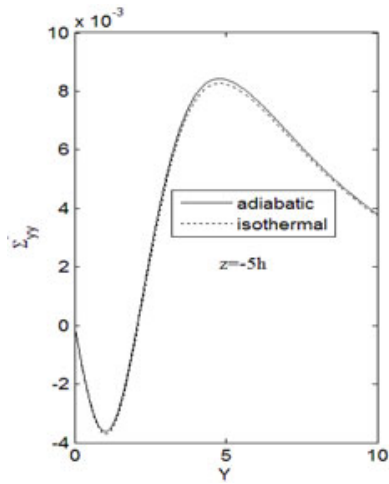

(b)

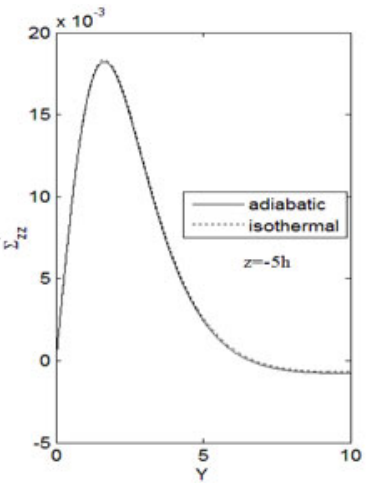

(c)

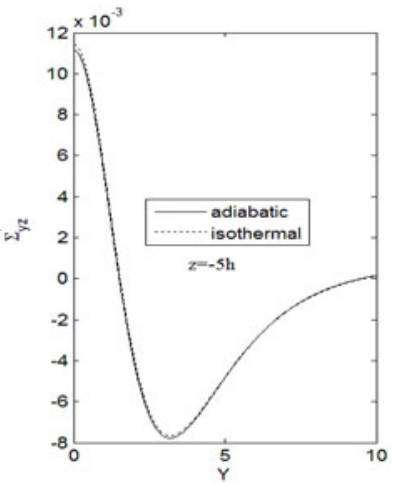

Fig. 23. Variation of the stresses $\Sigma_{y y}^{\prime}\left(\right.$ a), $\Sigma_{z z}^{\prime}$ (b), and $\Sigma_{y z}^{\prime}$ (c) at $z=-5 h$ with horizontal distance from the fault.

The effect of thermoelasticity in the deformation of elastic medium is more significant when the source is nearer to the interface $z=0$. If the elastic medium is considered as the crustal layer of the Earth and mantle layer is modeled as thermoelastic medium, then due to the fault occurring in the crust near the mantle, the effect of thermoelasticity is significant on the Earth's surface. 
List of parameters

\begin{tabular}{|c|c|}
\hline Symbol & Description \\
\hline$x, y, z$ & Cartesian coordinates \\
\hline$t$ & time \\
\hline$u_{i}$ & displacement components of thermoelastic medium \\
\hline$h$ & distance of the source from interface \\
\hline$\sigma_{i j}$ & stress tensor of thermoelastic medium \\
\hline$\varepsilon_{i j}$ & strain tensor of thermoelastic medium \\
\hline$\delta_{i j}$ & Kronecker delta \\
\hline$\lambda, \mu$ & Lame's constants of thermoelastic medium \\
\hline$\alpha_{t}$ & coefficient of linear thermal expansion \\
\hline$\beta=(3 \lambda+2 \mu) \alpha_{t}$ & thermoelastic coupling coefficient \\
\hline$\theta$ & $\begin{array}{l}\text { difference between absolute temperature and temperature } \\
\text { at the natural state }\end{array}$ \\
\hline$v$ & Poisson's ratio of thermoelastic medium \\
\hline$\lambda_{0}$ & thermal conductivity \\
\hline$C_{e}$ & specific heat \\
\hline$\rho$ & density \\
\hline$T_{0}$ & temperature of the medium at natural state \\
\hline$q_{i}$ & components of heat flux \\
\hline$\omega$ & frequency \\
\hline$u_{i}^{\prime}$ & displacement components of elastic medium \\
\hline$\mu^{\prime}$ & shear modulus of elastic medium \\
\hline$v^{\prime}$ & Poisson's ratio of elastic medium \\
\hline$\sigma_{i j}^{\prime}$ & stress tensor of elastic medium \\
\hline$\mu_{r}$ & ratio of rigidity of thermoelastic and elastic mediums \\
\hline$Y, Z$ & dimensionless Cartesian coordinates \\
\hline$T$ & dimensionless time \\
\hline$U_{i}^{\prime}$ & dimensionless displacement components \\
\hline$\Theta$ & dimensionless temperature difference \\
\hline$\Sigma_{i j}$ & dimensionless stress tensor of thermoelastic medium \\
\hline$\Sigma_{i j}^{\prime}$ & dimensionless stress tensor of elastic medium \\
\hline
\end{tabular}




\section{References}

Abd-Alla, A.M. (1995), Thermal stress in a transversely isotropic circular cylinder due to an instantaneous heat source, Appl. Math. Comput. 68, 2-3, 113-124, DOI: 10.1016/0096-3003(94)00085-I.

Ahrens, T.J. (ed.) (1995), Mineral Physics and Crystallography: A Handbook of Physical Constants, American Geophysical Union, Washington, D.C.

Aki, K., and P.G. Richards (1980), Quantitative Seismology: Theory and Methods, Vol. I and II, W.H. Freeman \& Co., San Francisco.

Attetkov, A.V., I.K. Volkov, and S.S. Pilyavskii (2009), Temperature field of a solid body containing a spherical heating source with a uniformly moving boundary, J. Eng. Phys. Thermophys. 82, 2, 368-375, DOI: 10.1007/ s10891-009-0185-x.

Ben-Menahem, A., and S.J. Singh (1981), Seismic Waves and Sources, 2nd ed., Springer Verlag, New York.

Burridge, R., and L. Knopoff (1964), Body force equivalents for seismic dislocations, Bull. Seismol. Soc. Am. 54, 6a, 1875-1888.

Dziewonski, A.M., and D.L. Anderson (1981), Preliminary reference Earth model, Phys. Earth Planet. In. 25, 4, 297-356, DOI: 10.1016/0031-9201(81)90046-7.

Freund, L.B., and D.M. Barnett (1976), A two-dimensional analysis of surface deformation due to dip-slip faulting, Bull. Seismol. Soc. Am. 66, 3, 667-675.

Garg, N.R., and S.J. Singh (1987), 2-D static response of a transversely isotropic multilayered half-space to surface loads, Indian J. Pure Appl. Math. 18, 8, 763-777.

Garg, N.R., D.K. Madan, and R.K. Sharma (1996), Two-dimensional deformation of an orthotropic elastic medium due to seismic sources, Phys. Earth Planet. In. 94, 1-2, 43-62, DOI: 10.1016/0031-9201(95)03095-6.

Garg, N.R., R. Kumar, A. Goel, and A. Miglani (2003), Plane strain deformation of an orthotropic elastic medium using an eigenvalue approach, Earth Planets Space 55, 1, 3-9, DOI: 10.1186/BF03352457.

Ghosh, M.K., and M. Kanoria (2007), Displacements and stresses in composite multi-layered media due to varying temperature and concentrated load, Appl. Math. Mech. 28, 6, 811-822, DOI: 10.1007/s10483-007-0611-5.

Heaton, T.H., and R.E. Heaton (1989), Static deformations from point forces and force couples located in welded elastic Poissonian half-spaces: Implications for seismic moment tensors, Bull. Seismol. Soc. Am. 79, 3, 813-841.

Hou, P.-F., A.Y.T. Leung, and C.-P. Chen (2008a), Fundamental solution for transversely isotropic thermoelastic materials, Int. J. Solids Struct. 45, 2, 392408, DOI: 10.1016/j.ijsolstr.2007.08.024.

Hou, P.-F., A.Y.T. Leung, and C.-P. Chen (2008b), Green's functions for semiinfinite transversely isotropic thermoelastic materials, ZAMM J. Appl. Math. Mech. 88, 1, 33-41, DOI: 10.1002/zamm.200710355. 
Hou, P.-F., L. Wang, and T. Yi (2009), 2D Green's functions for semi-infinite orthotropic thermoelastic plane, Appl Math. Model. 33, 3, 1674-1682, DOI: 10.1016/j.apm.2008.03.004.

Hou, P.-F., S. He, and C.-P. Chen (2011), 2D general solution and fundamental solution for orthotropic thermoelastic materials, Eng. Anal. Bound. Elem. 35, 1, 56-60, DOI: 10.1016/j.enganabound.2010.04.007.

Jovanovich, D.B., M.I. Husseini, and M.A. Chinnery (1974a), Elastic dislocations in a layered half-space - I. Basic theory and numerical methods, Geophys. J. Int. 39, 2, 205-217, DOI: 10.1111/j.1365-246X.1974.tb05451.x.

Jovanovich, D.B., M.I. Husseini, and M.A. Chinnery (1974b), Elastic dislocations in a layered half-space - II. The point source, Geophys. J. Int. 39, 2, 219-239, DOI: 10.1111/j.1365-246X.1974.tb05452.x.

Kit, H.S., B.E. Monastyrs'kyi, and O.P. Sushko (2001), Thermoelastic state of a semiinfinite body with plane surface crack under the action of heat sources, Mater. Sci. 37, 4, 610-614, DOI: 10.1023/A:1013272721094.

Kumar, A., S.J. Singh, and J. Singh (2005), Deformation of two welded elastic halfspaces due to a long inclined tensile fault, J. Earth Syst. Sci. 114, 1, 97-103, DOI: $10.1007 / \mathrm{BF} 02702012$.

Kumar, R., and R.R. Gupta (2009), Plane strain deformation in an orthotropic micropolar thermoelastic solid with a heat source, J. Eng. Phys. Thermophys. 82, 3, 556-565, DOI: 10.1007/s10891-009-0220-y.

Kumar, R., and L. Rani (2004), Deformation due to mechanical and thermal sources in generalised orthorhombic thermoelastic material, Sadhana 29, 5, 429447, DOI: 10.1007/BF02703254.

Kumari, G., S.J. Singh, and K. Singh (1992), Static deformation of two welded elastic half-spaces caused by a point dislocation source, Phys. Earth Planet. In. 73, 1-2, 53-76, DOI: 10.1016/0031-9201(92)90107-7.

Lanzano, P. (1986a), Thermoelastic deformations of Earth's lithosphere: A mathematical model, Earth Moon Planets 34, 3, 283-304, DOI: 10.1007/ BF00145087.

Lanzano, P. (1986b), Heat conduction within an elastic Earth, Earth Moon Planets 36, 2, 157-166, DOI: 10.1007/BF00057608.

Lay, T., and T.C. Wallace (1995), Modern Global Seismology, Academic Press, San Diego.

Madan, D.K., K. Singh, R. Aggarwal, and A. Gupta (2005), Displacements and stresses in an anisotropic medium due to non-uniform slip along a very long strike-slip fault, ISET J. Earthq. Technol. 42, 1, 1-11.

Mallik, S.H., and M. Kanoria (2008), A two dimensional problem for a transversely isotropic generalized thermoelastic thick plate with spatially varying heat source, Eur. J. Mech. A 27, 4, 607-621, DOI: 10.1016/j.euromechsol.2007. 09.002.

Maruyama, T. (1964), Statical elastic dislocations in an infinite and semi-infinite medium, Bull. Earthq. Res. Inst. Univ. Tokyo 42, 2, 289-368. 
Maruyama, T. (1966), On two-dimensional elastic dislocations in an infinite and semi-infinite medium, Bull. Earthq. Res. Inst. Univ. Tokyo 44, 811-871.

Nowacki, W. (1975), Dynamical Problems of Thermoelasticity, PWN Polish Sci. Publ., Warszawa, Noordhoff Int. Publ., Leyden.

Okada, Y. (1985), Surface deformation due to shear and tensile faults in a halfspace, Bull. Seismol. Soc. Am. 75, 4, 1135-1154.

Okada, Y. (1992), Internal deformation due to shear and tensile faults in a halfspace, Bull. Seismol. Soc. Am. 82, 2, 1018-1040.

Pan, E. (1989a), Static response of a transversely isotropic and layered half-space to general surface loads, Phys. Earth Planet. In. 54, 3-4, 353-363, DOI: 10.1016/0031-9201(89)90252-5.

Pan, E. (1989b), Static response of a transversely isotropic and layered half-space to general dislocation sources, Phys. Earth Planet. In. 58, 2-3, 103-117, DOI: 10.1016/0031-9201(89)90046-0.

Pan, E. (1990), Thermoelastic deformation of a transversely isotropic and layered half-space by surface loads and internal sources, Phys. Earth Planet. Int. 60, 1-4, 254-264, DOI: 10.1016/0031-9201(90)90266-Z.

Rani, S., S.J. Singh, and N.R. Garg (1991), Displacements and stresses at any point of a uniform half-space due to two-dimensional buried sources, Phys. Earth Planet. Int. 65, 3-5, 276-282, DOI: 10.1016/0031-9201(91)90134-4.

Rongved, L., and J.T. Frasier (1958), Displacement discontinuity in the elastic halfspace, J. Appl. Mech. 25, 125-128.

Rundle, J.B. (1982), Some solutions for static and pseudo-static deformation in layered, nonisothermal, porous media, J. Phys. Earth 30, 5, 421-440, DOI: 10.4294/jpe1952.30.421.

Sato, R. (1971), Crustal deformation due to dislocation in a multi-layered medium, J. Phys. Earth 19, 1, 31-46, DOI: 10.4294/jpe1952.19.31.

Sato, R., and M. Matsu'ura (1973), Static deformations due to the fault spreading over several layers in a multi-layered medium. Part I: Displacement, J. Phys. Earth 21, 3, 227-249, DOI: 10.4294/jpe1952.21.227.

Schapery, R.A. (1962), Approximate methods of transform inversion for viscoelastic stress analysis. In: Proc. 4th U.S. National Congress of Applied Mechanics, 18-21 June 1962, Berkeley USA, Vol. 2, 1075-1085, American Society of Mechanical Engineers, New York.

Shevchenko, V.P., and A.S. Gol'tsev (2001), The thermoelastic state of orthotropic shells heated by concentrated heat sources, Int. Appl. Mech. 37, 5, 654-661, DOI: $10.1023 / \mathrm{A}: 1012364530719$.

Singh, K., D.K. Madan, A. Goel, and N.R. Garg (2005), Two-dimensional static deformation of an anisotropic medium, Sadhana 30, 4, 565-583, DOI: 10.1007/BF02703280.

Singh, S.J. (1970), Static deformation of a multilayered half-space by internal sources, J. Geophys. Res. 75, 17, 3257-3263, DOI: 10.1029/ JB075i017p03257. 
Singh, S.J., and A. Ben-Menahem (1969), Displacement and strain fields due to faulting in a sphere, Phys. Earth Planet. In. 2, 2, 77-87, DOI: 10.1016/ 0031-9201(69)90003-X.

Singh, S.J., and N.R Garg (1985), On two-dimensional elastic dislocations in a multilayered half-space, Phys. Earth Planet. In. 40, 2, 135-145, DOI: 10.1016/ 0031-9201(85)90067-6.

Singh, S.J., and N.R. Garg (1986), On the representation of two-dimensional seismic sources, Acta Geophys. Pol. 34, 1, 1-12.

Singh, S.J., A. Ben-Menahem, and M. Vered (1973), A unified approach to the representation of seismic sources, Proc. Roy. Soc. London A 331, 1587, 525551, DOI: $10.1098 /$ rspa.1973.0006.

Singh, S.J., S. Rani, and N.R. Garg (1992), Displacements and stresses in two welded half-spaces caused by two-dimensional sources, Phys. Earth Planet. In. 70, 1, 90-101, DOI: 10.1016/0031-9201(92)90164-Q.

Singh, S.J., G. Kumari, and K. Singh (1993), Static deformation of two welded elastic half-spaces caused by a finite rectangular fault, Phys. Earth Planet. In. 79, 3, 313-333, DOI: 10.1016/0031-9201(93)90112-M.

Singh, S.J., A. Kumar, and J. Singh (2003), Deformation of a monoclinic elastic half-space by a long inclined strike-slip fault, ISET J. Earthq. Technol. 40, 1, 51-59.

Small, J.C., and J.R. Booker (1986), The behaviour of layered soil or rock containing a decaying heat source, Int. J. Numer. Anal. Meth. Geomech. 10, 5, 501519, DOI: $10.1002 /$ nag.1610100504.

Stein, S., and M. Wysession (2003), An Introduction to Seismology, Earthquakes, and Earth Structure, Blackwell Publishing, Oxford.

Steketee, J.A. (1958), On Volterra's dislocations in a semi-infinite elastic medium, Can. J. Phys. 36, 2, 192-205, DOI: 10.1139/p58-024.

Tomar, S.K., and N.K. Dhiman (2003), 2-D deformation analysis of a half-space due to a long dip-slip fault at finite depth, J. Earth Syst. Sci. 112, 4, 587-596, DOI: $10.1007 /$ BF02709782.

Youssef, H.M. (2006), Problem of generalized thermoelastic infinite medium with cylindrical cavity subjected to a ramp-type heating and loading, Arch. Appl. Mech. 75, 8-9, 553-565, DOI: 10.1007/s00419-005-0440-3.

Youssef, H.M. (2009), Generalized thermoelastic infinite medium with cylindrical cavity subjected to moving heat source, Mech. Res. Commun. 36, 4, 487496, DOI: 10.1016/j.mechrescom.2008.12.004.

Youssef, H.M. (2010), Two-temperature generalized thermoelastic infinite medium with cylindrical cavity subjected to moving heat source, Arch. Appl. Mech. 80, 11, 1213-1224, DOI: 10.1007/s00419-009-0359-1.

Received 7 January 2014 Received in revised form 10 June 2014 